\title{
SPECTRAL ENERGY DISTRIBUTIONS OF TYPE 1 ACTIVE GALACTIC NUCLEI IN THE COSMOS SURVEY. I. THE XMM-COSMOS SAMPLE
}

\author{
M. Elvis ${ }^{1}$, H. HaO ${ }^{1,2}$, F. Civano ${ }^{1}$, M. Brusa ${ }^{3}$, M. Salvato ${ }^{3,4,5}$, A. Bongiorno ${ }^{3,6}$, P. Capak ${ }^{7}$ G. Zamorani $^{8}$, A. Comastri $^{8}$, \\ K. Jahnke ${ }^{9}$, E. Lusso ${ }^{9}$, V. Mainieri ${ }^{10}$, J. R. Trump ${ }^{11,12}$, L. C. Ho ${ }^{13}$, H. Aussel ${ }^{14}$, N. Cappelluti ${ }^{3,8}$, M. Cisternas $^{9,15}$, \\ D. Frayer ${ }^{16}$, R. Gilli ${ }^{8}$, G. Hasinger ${ }^{17}$, J. P. Huchra ${ }^{1,30}$, C. D. ImPeY ${ }^{11}$, A. M. KoeKemoer ${ }^{18}$, G. Lanzuisi ${ }^{1,3,19,20}$, \\ E. Le Floc' ${ }^{21}$, S. J. Lilly ${ }^{22}$, Y. Liu ${ }^{23}$, P. McCarthy ${ }^{13}$, H. J. McCracken ${ }^{24}$, A. Merloni ${ }^{3}$, H.-J. Roeser ${ }^{9}$, D. B. Sanders ${ }^{17}$, \\ M. Sargent ${ }^{9,21}$, N. Scoville ${ }^{7}$, E. Schinnerer ${ }^{9}$, D. Schiminovich ${ }^{25}$, J. Silverman ${ }^{26}$, Y. TANiguchi ${ }^{27}$, C. Vignali $^{28}$, \\ C. M. Urry ${ }^{29}$, M. A. Zamojski ${ }^{25}$, And M. Zatloukal ${ }^{9}$ \\ ${ }^{1}$ Harvard Smithsonian Center for astrophysics, 60 Garden St., Cambridge, MA 02138, USA; \\ elvis@cfa.harvard.edu, hhao@cfa.harvard.edu \\ ${ }^{2}$ SISSA, Via Bonomea 265, I-34136 Trieste, Italy \\ ${ }^{3}$ Max-Planck-Institut für Extraterrestrische Physik, Postfach 1312, D-85741, Garching bei München, Germany \\ ${ }^{4}$ IPP-Max-Planck-Institut for Plasma Physics, Boltzmann Strasse 2, D-85748, Garching bei München, Germany \\ ${ }^{5}$ Excellence Cluster, Boltzmann Strasse 2, D-85748, Garching bei München, Germany \\ ${ }^{6}$ INAF-Osservatorio Astronomico di Roma, Via di Frascati 33, I-00040, Monteporzio Catone, Rome, Italy \\ ${ }^{7}$ California Institute of Technology, MC 105-24, 1200 East California Boulevard, Pasadena, CA 91125, USA \\ ${ }^{8}$ INAF-Osservatorio Astronomico di Bologna, via Ranzani 1, I-40127 Bologna, Italy \\ ${ }^{9}$ Max-Planck-Institut für Astronomie, Königstuhl 17, Heidelberg, D-69117, Germany \\ ${ }^{10}$ European Southern Observatory, Karl-Schwarzschild-Strasse 2, D-85748, Garching bei München, Germany \\ ${ }^{11}$ Steward Observatory, University of Arizona, 933 North Cherry Avenue, Tucson, AZ 85721, USA \\ $12 \mathrm{UCO} /$ Lick Observatory, University of California, Santa Cruz, CA 95064, USA \\ ${ }^{13}$ The Observatories of the Carnegie Institute for Science, Santa Barbara Street, Pasadena, CA 91101, USA \\ ${ }^{14}$ AIM Unité Mixte de Recherche CEA CNRS, Université Paris VII UMR n158, Paris, France \\ ${ }^{15}$ Instituto de Astrofísica de Canarias, E-38205, La Laguna, Tenerife, Spain \\ ${ }^{16}$ National Radio Astronomy Observatory, P.O. Box 2, Green Bank, WV 24944, USA \\ ${ }^{17}$ Institute for Astronomy, University of Hawaii, 2680 Woodlawn Drive, Honolulu, HI 96822, USA \\ ${ }^{18}$ Space Telescope Science Institute, 3700 San Martin Drive, Baltimore, MD 21218, USA \\ ${ }^{19}$ INAF-IASF Roma, Via Fosso del Cavaliere 100, I-00133 Rome, Italy \\ ${ }^{20}$ INAF-IASF Bologna, Via Gobetti 101, I-40129 Bologna, Italy \\ ${ }^{21}$ CEA-Saclay, Service d'Astrophysique, Orme des Merisiers, Bat. 709, F-91191 Gif-sur-Yvette, France \\ ${ }^{22}$ Institute of Astronomy, Swiss Federal Institute of Technology (ETH Hönggerberg), CH-8093, Zürich, Switzerland \\ ${ }^{23}$ Key Laboratory of Particle Astrophysics, Institute of High Energy Physics, Chinese Academy of Sciences, P.O. Box 918-3, Beijing 100049, China \\ ${ }^{24}$ Institut d'Astrophysique de Paris, UMR 7095 CNRS, Université Pierre et Marie Curie, 98 bis Boulevard Arago, F-75014 Paris, France \\ 25 Department of Astronomy, Columbia University, MC2457, 550 W. 120 St., New York, NY 10027, USA \\ ${ }^{26}$ Institute for the Physics and Mathematics of the Universe (IPMU), University of Tokyo, Kashiwanoha 5-1-5, Kashiwa-shi, Chiba 277-8568, Japan \\ ${ }^{27}$ Research Center for Space and Cosmic Evolution, Ehime University, Bunkyo-cho 2-5, Matsuyama 790-8577, Japan \\ ${ }^{28}$ Dipartimento di Astronomia, Università degli Studi di Bologna, via Ranzani 1, I-40127 Bologna, Italy \\ ${ }^{29}$ Physics Department and Yale Center for Astronomy and Astrophysics, Yale University, New Haven, CT 06511, USA \\ Received 2011 December 14; accepted 2012 September 4; published 2012 October 10
}

\begin{abstract}
The "Cosmic Evolution Survey" (COSMOS) enables the study of the spectral energy distributions (SEDs) of active galactic nuclei (AGNs) because of the deep coverage and rich sampling of frequencies from X-ray to radio. Here we present an SED catalog of 413 X-ray (XMM-Newton)-selected type 1 (emission line FWHM $>2000 \mathrm{~km} \mathrm{~s}^{-1}$ ) AGNs with Magellan, SDSS, or VLT spectrum. The SEDs are corrected for Galactic extinction, broad emission line contributions, constrained variability, and host galaxy contribution. We present the mean SED and the dispersion SEDs after the above corrections in the rest-frame $1.4 \mathrm{GHz}$ to $40 \mathrm{keV}$, and show examples of the variety of SEDs encountered. In the near-infrared to optical (rest frame $\sim 8 \mu \mathrm{m}-4000 \AA$ ), the photometry is complete for the whole sample and the mean SED is derived from detections only. Reddening and host galaxy contamination could account for a large fraction of the observed SED variety. The SEDs are all available online.
\end{abstract}

Key words: galaxies: evolution - quasars: general - surveys

Online-only material: color figures, supplemental data (FITS) file (tar.gz)

\section{INTRODUCTION}

Quasars and active galactic nuclei (AGNs) are the most luminous persistent sources of radiation in the universe. The AGN luminosity is emitted primarily in a broad continuum spectrum that carries significant power over several decades, from the far-infrared (FIR) to the X-ray bands. Hence, knowing the spectral energy distributions (SEDs) of AGNs is essential to a deeper understanding of quasar physics. The mean SED

\footnotetext{
${ }_{30}$ John P. Huchra has contributed to the work before his death in 2010 October.
}

compiled by Elvis et al. (1994, E94 hereinafter) is still the most commonly used SED for quasars, despite recent additions and updates (see e.g., Polletta et al. 2000; Kuraszkiewicz et al. 2003; Marconi et al. 2004; Risaliti \& Elvis 2004; Richards et al. 2006; Hopkins et al. 2006; Polletta et al. 2007; Shang et al. 2011; Luo et al. 2010; Lusso et al. 2010; Marchese et al. 2012; Trichas et al. 2012). However, the E94 SEDs suffer from significant limitations: (1) the sample is primarily an ultraviolet-excessselected sample, and is further biased toward relatively X-ray loud quasars; (2) the mean SED was compiled from a small 
number of AGNs (29 radio-quiet and 18 radio-loud AGNs); (3) the sample only covers a low redshift range $(0.05 \leqslant z \leqslant 0.9$, with $80 \%$ being at $z<0.3$ ); (4) the data in the X-ray, ultraviolet, and far-infrared region have limited signal-to-noise ratios (S/ Ns), with a large number of upper limits. Even so, E94 found a large dispersion in SEDs of $\sim 1$ dex at both $100 \mu \mathrm{m}$ and $0.1 \mu \mathrm{m}$, when all the SEDs were normalized at $1 \mu \mathrm{m}$. The $1 \mu \mathrm{m}$ wavelength is usually chosen as a normalization point because it is the approximate location of the inflection between the rising Wien tail of emission from hot dust and the power-law $f_{v} \propto v^{1 / 3}$ of the big blue bump in the optical caused by the emission of the accretion disk in $v f_{v}$ versus $v$ space (E94). This variety of continuum shapes in quasars has not been carefully explored so that no correlations of SED shape in the FIR-UV with other properties have been found, nor is there an accepted theoretical explanation fitting all the various forms.

The Cosmic Evolution Survey (COSMOS; Scoville et al. $2007 b$ ) has the appropriate combination of depth, area and multiwavelength coverage that allows detection of substantial AGN samples by all standard techniques-X-ray (Brusa et al. 2007, 2010; Civano et al. 2012), infrared (Donley et al. 2012), radio (Schinnerer et al. 2010), and optical (Gabor et al. 2009). COSMOS covers a $2 \mathrm{deg}^{2}$ equatorial field centered on R.A. = 10:00:28.6, Decl. = +02:12:21 (J2000) with deep imaging by most of the major space-based telescopes: Hubble (Scoville et al. 2007a; Koekemoer et al. 2007), Spitzer (Sanders et al. 2007), GALEX (Zamojski et al. 2007), XMM-Newton (Hasinger et al. 2007; Cappelluti et al. 2009), and Chandra (Elvis et al. 2009), and large ground-based telescopes: Subaru (Taniguchi et al. 2007), VLA (Schinnerer et al. 2007, 2010), CFHT (McCracken et al. 2007), and UKIRT, NOAO (Capak et al. 2007). The imaging coverage is complemented by dedicated redshift surveys conducted with the VIMOS/VLT (zCOSMOS; Lilly et al. 2007, 2009), IMACS/Magellan (Trump et al. 2007, 2009a), and DEIMOS/Keck, ${ }^{31}$ for a total of more than $20 \mathrm{k}$ spectra collected. The field has also been covered by SDSS (Abazajian et al. 2004), for both photometry and spectroscopy.

COSMOS contains over 400 type 1 (i.e., broad emission line, FWHM $>2000 \mathrm{~km} \mathrm{~s}^{-1}$ ) AGNs, spanning a wide range of redshifts, that were identified through the MMT/IMACS, zCOSMOS, and SDSS surveys (Brusa et al. 2007, 2010). So, far the COSMOS data set has 43 photometric bands, with high $\mathrm{S} / \mathrm{N}(\gtrsim 80)$ for a typical $I=22.5$ type 1 AGN. Hence, COSMOS overcomes the limitations of E94, offering an opportunity to make order of magnitude improvements in our knowledge of AGN SEDs.

This is the first of a series of papers on the SEDs of the X-ray-selected type 1 AGN in COSMOS. The main goal of this study is to improve the type 1 AGN SED template over that of E94 and, perhaps, more importantly, to study the diversity of AGN SEDs and their relation to physical parameters. Lusso et al. (2010), in a complementary paper, analyzed a sample of 545 XMM-Newton-selected type 1 AGNs in $X M M$-COSMOS, including both the spectroscopic $(361 / 545)$ and photometric $(184 / 545)$ identifications in order to study dependence on luminosity and redshift of the relationship between the UV and X-ray luminosity $\left(\alpha_{\mathrm{OX}}\right)$. Trump et al. (2011) also presented SED information for 348 XMM-Newton-selected AGNs in COSMOS, although they focused on the differences between type 1 and unobscured type 2 AGN SEDs rather than

\footnotetext{
31 The result of a multi-year observing campaign (PIs: Capak, Kartaltepe, Salvato, Sanders, Scoville; see Kartaltepe et al. 2010).
}

the dispersion among type 1 AGNs themselves. This paper (Paper I) describes the properties of the sample used for this study and presents the mean SED, including corrections for the Galactic extinction, broad emission line (BEL) contribution, while limiting both variability and host galaxy contamination. Three other companion papers on this subject are in preparation (Hao et al. 2012a, 2012b, 2012c). The companion Paper II (Hao et al. 2012a) investigates the systematic trends in the shapes of the SED in the wavelength range of $0.3-3 \mu \mathrm{m}$ as a function of redshift, bolometric luminosity, black hole mass, and Eddington ratio. Paper III (Hao et al. 2012b) introduces a "mixing diagram" (near-infrared SED slope versus optical SED slope plot) to understand the diversity of the SED shapes in XMM-COSMOS type 1 AGN sample, provide a new estimation of the host galaxy fraction and reddening, and identify interesting outliers. Paper IV (Hao et al. 2012c) studies the radio-loudness of the $X M M$-COSMOS type 1 AGN. An interesting subsample of "hot dust poor" quasars, drawn from this sample, have already been presented in Hao et al. (2010).

In this paper, all magnitudes are reported in the $\mathrm{AB}$ system (Oke \& Gunn 1983) and the Wilkinson Microwave Anisotropy Probe 5 year cosmology (Komatsu et al. 2009), with $H_{0}=$ $71 \mathrm{~km} \mathrm{~s}^{-1} \mathrm{Mpc}^{-1}, \Omega_{M}=0.26$ and $\Omega_{\Lambda}=0.74$ assumed.

\section{SAMPLE DESCRIPTION}

$\mathrm{X}$-ray emission is ubiquitous in AGNs, and in the X-ray band, the obscuration and complication from host galaxy light are minimized. Hence, X-ray surveys give the most complete and effective census of AGNs of any single band (Risaliti \& Elvis 2004). The XMM-COSMOS survey (Hasinger et al. 2007) observed the entire COSMOS field for a total of $\sim 1.5 \mathrm{Ms}$, with an average exposure of $60 \mathrm{ks}$ across the field to a depth of $\sim 5 \times 10^{-16} \mathrm{erg} \mathrm{cm}^{-2} \mathrm{~s}^{-1}(0.5-2 \mathrm{keV})$ and $\sim 3 \times 10^{-15} \mathrm{erg} \mathrm{cm}^{-2} \mathrm{~s}^{-1}(2-10 \mathrm{keV})$ over $90 \%$ of the surveyed area. As such XMM-COSMOS is the deepest survey over such a large contiguous solid angle $\left(2.13 \mathrm{deg}^{2}\right)$ performed to date with XMM-Newton (Cappelluti et al. 2009). X-ray fluxes were computed from the count rates assuming the Galactic column density of $N_{\mathrm{H}}=2.6 \times 10^{20} \mathrm{~cm}^{-2}$ (Dickey \& Lockman 1990) and spectral indices of $\Gamma=2$ for the $0.5-2 \mathrm{keV}$ band and $\Gamma=1.7$ for the $2-10 \mathrm{keV}$ band (Cappelluti et al. 2009). A total of 1848 point-like sources were detected: 1567 in the $0.5-2 \mathrm{keV}, 1096$ in the $2-8 \mathrm{keV}$ band, and 245 in the $4.5-10 \mathrm{keV}$ band, excluding those associated with extended X-ray sources.

Brusa et al. (2007, 2010) used the likelihood ratio technique (Sutherland \& Saunders 1992; Ciliegi et al. 2003; Brusa et al. 2005; Civano et al. 2012) to identify the counterparts of XMM-COSMOS sources using the optical (CFHT $I$ band), near-infrared (CFHT $K$ band), and mid-infrared (IRAC) catalogs. For the subfield of the XMM-COSMOS also covered by Chandra, the optical identifications have been augmented with the more accurate Chandra positions (Civano et al. 2012). $85 \%$ (1577) of the XMM-Newton sources have a unique and secure optical counterpart with a probability of misidentification of $<0.01$ (i.e., no ambiguous optical counterparts, no XMMNewton sources separated into two sources in the Chandra cata$\log$; see Table 1 of Brusa et al. 2010 for details). The counterparts have been cross-correlated with the full COSMOS photometric catalog (Capak et al. 2007), including optical bands from CFHT, SDSS, and Subaru multiwavelength bands (Taniguchi et al. 2007), which consists of 6 broad $(\Delta \lambda=800-1500 \AA)$ bands, 12 intermediate $(\Delta \lambda=300-500 \AA)$ bands, and 2 narrow $(\Delta \lambda=150-200 \AA)$ bands. The counterparts have also been 
cross-correlated with the CFHT J-band (Ilbert et al. 2009), CFHT $K$-band (McCracken et al. 2010) catalog. The sources were also associated with the Galaxy Evolution Explorer (GALEX) sources from the deblended, PSF-fitted GALEX COSMOS catalog (Zamojski et al 2007), and with the IRAC (Sanders et al. 2007), the MIPS $24 \mu \mathrm{m}$ (Le Floc'h et al. 2009), and the $70 \mu \mathrm{m}$ (Frayer et al. 2009; Kartaltepe et al. 2010) catalogs. More details on the cross-correlation procedure and source identification are available in Brusa et al. (2010).

The counterpart positions were then cross-correlated with all the available redshift catalogs in the field, including the most recent SDSS release (Schneider et al. 2007), the COSMOS AGN spectroscopic survey, and the zCOSMOS survey catalogs. The Magellan/IMACS AGN spectroscopic survey has optical spectra of an X-ray and optical flux-limited sample (down to $f_{0.5-10 \mathrm{keV}}>8 \times 10^{-16} \mathrm{erg} \mathrm{cm}^{-2} \mathrm{~s}^{-1}$ and $\left.i_{\mathrm{AB}}^{+}<23\right)$ of 677 XMM-Newton-selected sources over the entire COSMOS field. Trump et al. (2009a) find 485 high-confidence redshifts from the first 3 years of the Magellan/IMACS survey, with a total of 588 AGNs with high-confidence redshifts at the survey's completion. zCOSMOS is a redshift survey of 20,000 galaxies in the COSMOS field taken with the VLT/VIMOS spectrograph. The zCOSMOS bright sample is magnitudelimited with $i_{\mathrm{AB}}^{+}<22.5$. XMM-Newton source counterparts were targeted explicitly. About 500 XMM-Newton sources have zCOSMOS spectra (many of which also have IMACS spectra). In summary, a total of 886 unique good quality spectroscopic redshifts are available, or $\sim 50 \%$ of the entire XMM-COSMOS sample (see details in Brusa et al. 2010). From this sample, we selected all the objects identified as type 1 AGNs (i.e., those showing BELs with FWHM $>2000 \mathrm{~km} \mathrm{~s}^{-1}$ in their optical spectra). This gives the final sample of 413 type $1 \mathrm{XMM}$ COSMOS AGNs: the "XC413" sample.

The near-infrared data set of COSMOS so far has only the $J$ and $K$ bands, leading to rather sparse coverage of the rest-frame optical parts of the SEDs for redshifts $\sim 1-2$. $H$-band imaging of the COSMOS field to $H_{\mathrm{AB}} \sim 21$ has been obtained as part of the Heidelberg InfraRed/Optical Cluster Survey (HIROCS survey; Falter et al. 2004). $H$-band total magnitudes (obtained through point-spread function (PSF) fitting) for 218 type 1 AGNs in this sample were supplied to us (H.-J. Röser 2010, private communication).

$1.4 \mathrm{GHz}$ counterparts to the X-ray sources were determined by positional matching the optical coordinates of the X-ray sources to the positions in the VLA-COSMOS Joint catalog (Schinnerer et al. 2010) with a search radius of $1^{\prime \prime}$. The VLACOSMOS joint catalog lists 2900 sources detected at $\mathrm{S} / \mathrm{N} \geqslant$ 5 in the COSMOS field. All successful matches (61 sources) were unique. The AIPS/MAXFIT peak finding algorithm was used to search for additional radio detections within a 2 .'5 $\times$ 2 '. 5 box centered on the optical coordinates of unmatched X-ray sources. For the 78 detections in the range $3 \sigma-5 \sigma$, we computed their total flux using the assumption that they are not resolved at $1.4 \mathrm{GHz}$ (beam FWHM 2'.5). For flux peaks with a lower significance level we derived a $3 \sigma$ upper limit based on the local rms noise (calculated within a $17^{\prime \prime} .5 \times 17^{\prime \prime} .5$ box) at the position of the radio source.

To derive SEDs for the XC413, we made use of all the data described above. Overall, the photometry is complete in 30 bands for more than $\sim 99 \%$ of the XC413 sample, and it is complete in five additional bands for more than $\sim 90 \%$ of the sample. In the infrared to ultraviolet wavelength range, where the bulk of the AGN power is radiated, we have complete

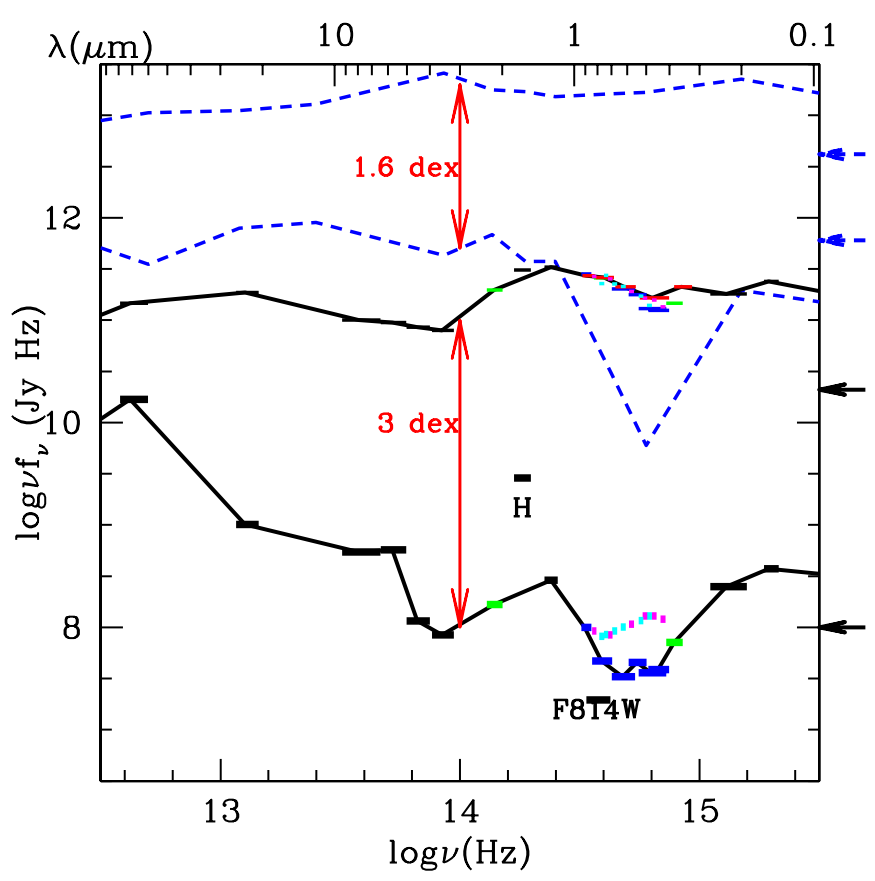

Figure 1. Spectral window function of the 413 type 1 AGNs in $X M M$-COSMOS (XC413, black solid line) compared to the E94 (blue dashed line) sample. The spectral window function consists of two lines. The upper line shows the brightest data and the lower line shows the limits of each band. From left to right the points are MIPS70, MIPS24, IRAC4-IRAC1 (black), CFHT $K$ band (green), $H$ band (black), $J$ band (black), ACS F814W band (black), Subaru broad bands (blue), SDSS (red), Subaru intermediate bands (2006 magenta, 2007 cyan), CFHT $u$ band (green), and GALEX NUV and FUV bands (black). The X-ray window width at $2 \mathrm{keV}$ is shown as arrows on the right.

(A color version of this figure is available in the online journal.)

coverage in the six infrared bands (IRAC $1-4, J$, and $K$ ), and complete, richer, coverage in the optical (Subaru broad and intermediate bands). This makes the XC413 sample the best so far with which to study the infrared to optical SED in great detail. Table 1 reports the bands used, their effective wavelengths and filter widths, the limiting depth of the survey, the number of XC413 sources having a detection in each band, and the observation dates. For convenience we also tabulate the value of $\mathrm{A}_{\lambda}$ for the typical Galactic extinction and the coefficient, $k_{\lambda}$, used for each band to calculate the Galactic extinction.

\section{SAMPLE PROPERTIES}

The depth of the multiwavelength coverage in COSMOS results in a wide range of detectable fluxes at most wavelengths. In Figure 1, we show the COSMOS "spectral window function" (Brissenden 1989) over the infrared to ultraviolet bands of $\mathrm{XC} 413$ in $\log v f_{v}$ versus $\log v$ space. The spectral window function is composed of two curves: the upper one picks out the brightest objects in the XC413 sample in each band; the lower one shows the limiting flux for detection in each band. The spectral window function thus shows the range of SEDs detectable for a sample. The width of the window function in the X-ray band is also indicated with arrows on the right. The XC413 AGN spectral window function has a typical width of $\sim 3$ dex in the infrared-optical-ultraviolet part of the spectrum where the bulk of the AGN power is radiated. This is about twice the range in the E94 sample (dashed blue lines) in almost all of the bands. Hence, the XC413 sample is capable of revealing a larger variety of type 1 AGN SEDs than the E94 sample (see Section 4.5 for details). 
Table 1

Data Quality and Depth

\begin{tabular}{|c|c|c|c|c|c|c|c|c|}
\hline $\begin{array}{l}\text { Filter } \\
\text { Name }\end{array}$ & Telescope & $\begin{array}{c}\text { Effective }^{\mathrm{a}} \\
\text { Wavelength }(\AA)\end{array}$ & $\begin{array}{c}\text { Filter }^{\mathrm{b}} \\
\text { Width }(\AA)\end{array}$ & Depth $^{\mathrm{c}}$ & $\begin{array}{l}\text { Number of } \\
\text { Detections }\end{array}$ & $\begin{array}{c}\text { Observations } \\
\text { Date }\end{array}$ & $k_{\lambda}^{\mathrm{d}}$ & $\overline{A_{\lambda}}{ }^{\mathrm{e}}$ \\
\hline $\bar{X}$ & $X M M$ & $2 \mathrm{keV}$ & $0.5-10 \mathrm{keV}$ & $10^{-15 * *}$ & 413 & 2003 Dec-2005 May & & \\
\hline$F U V$ & GALEX & 1540 & 209 & 25.69 & 136 & $2004 \mathrm{Feb}$ & 8.92 & 0.17 \\
\hline$N U V$ & GALEX & 2315 & 797 & 25.99 & 263 & $2004 \mathrm{Feb}$ & 7.97 & 0.15 \\
\hline$u$ & SDSS & 3564 & 600 & 22.00 & 370 & $2001 \mathrm{Jul}^{*}$ & 4.69 & 0.09 \\
\hline$u^{*}$ & CFHT & 3823 & 605 & 26.50 & 412 & 2003 Jan-2007 Apr & 4.69 & 0.09 \\
\hline IA427 & Subaru & 4263 & 208 & 25.82 & 411 & 2006 Jan & 4.21 & 0.08 \\
\hline$B_{J}$ & Subaru & 4458 & 897 & 27.00 & 413 & 2004 Jan & 4.04 & 0.08 \\
\hline I A464 & Subaru & 4635 & 218 & 25.65 & 411 & $2006 \mathrm{Feb}$ & 3.86 & 0.07 \\
\hline$g$ & SDSS & 4723 & 1300 & 22.20 & 405 & $2001 \mathrm{Jul}^{*}$ & 3.74 & 0.07 \\
\hline$g^{+}$ & Subaru & 4777 & 1265 & 27.00 & 413 & 2004 Feb & 3.74 & 0.07 \\
\hline I A484 & Subaru & 4849 & 229 & 25.60 & 410 & 2007 Jan & 3.67 & 0.07 \\
\hline I A505 & Subaru & 5063 & 232 & 25.55 & 412 & $2006 \mathrm{Feb}$ & 3.45 & 0.07 \\
\hline I A527 & Subaru & 5261 & 243 & 25.62 & 410 & 2007 Jan & 3.29 & 0.06 \\
\hline$V_{J}$ & Subaru & 5478 & 946 & 26.60 & 412 & $2004 \mathrm{Feb}$ & 3.15 & 0.06 \\
\hline I A574 & Subaru & 5765 & 273 & 25.61 & 412 & 2006 Jan & 2.96 & 0.06 \\
\hline$r$ & SDSS & 6202 & 1200 & 22.00 & 401 & $2001 \mathrm{Jul}^{*}$ & 2.59 & 0.05 \\
\hline I A624 & Subaru & 6233 & 300 & 25.60 & 410 & 2006 Dec & 2.61 & 0.05 \\
\hline$r^{+}$ & Subaru & 6289 & 1382 & 26.80 & 413 & 2004 Jan & 2.59 & 0.05 \\
\hline I A679 & Subaru & 6781 & 336 & 25.60 & 412 & 2006 Feb & 2.27 & 0.04 \\
\hline I A709 & Subaru & 7074 & 317 & 25.65 & 411 & 2006 Jan & 2.15 & 0.04 \\
\hline NB711 & Subaru & 7120 & 73 & 25.00 & 412 & $2006 \mathrm{Feb}$ & 2.13 & 0.04 \\
\hline I A738 & Subaru & 7362 & 324 & 25.60 & 410 & 2007 Jan & 2.04 & 0.04 \\
\hline$i$ & SDSS & 7523 & 1300 & 21.30 & 406 & $2001 \mathrm{Jul}^{*}$ & 1.92 & 0.04 \\
\hline$i^{*}$ & CFHT & 7618 & 1300 & 24.00 & 411 & 2004 Jan & 1.92 & 0.04 \\
\hline$i^{+}$ & Subaru & 7684 & 1495 & 26.20 & $199^{\mathrm{f}}$ & 2004 Jan & 1.92 & 0.04 \\
\hline I A767 & Subaru & 7685 & 364 & 25.60 & 412 & 2007 Mar & 1.92 & 0.04 \\
\hline$F 814 W$ & $H S T$ & 8072 & 1830 & 27.10 & $388^{\mathrm{g}}$ & 2003 Oct* & 1.80 & 0.03 \\
\hline N B816 & Subaru & 8149 & 120 & 25.70 & 413 & 2005 Feb* $^{*}$ & 1.74 & 0.03 \\
\hline I A827 & Subaru & 8245 & 343 & 25.39 & 411 & 2006 Jan & 1.69 & 0.03 \\
\hline$z$ & SDSS & 8905 & 1000 & 20.50 & 368 & $2001 \mathrm{Jul}^{*}$ & 1.44 & 0.03 \\
\hline$z^{+}$ & Subaru & 9037 & 856 & 25.20 & 411 & 2004 Jan & 1.44 & 0.03 \\
\hline$J$ & UH $88^{\prime \prime}$ & 12491 & 1580 & 23.70 & 413 & 2006 Mar & 0.97 & 0.02 \\
\hline$H$ & Calar Alto & 16483 & 2665 & 20.90 & 252 & 2005 Aug & & \\
\hline$K$ & KPNO & 21537 & 3120 & 21.60 & 406 & 2004 Feb* $^{*}$ & 0.34 & 0.01 \\
\hline$K$ & CFHT & 21590 & 3255 & 23.70 & 413 & 2007 Mar & 0.34 & 0.01 \\
\hline$I R A C 1$ & Spitzer & 35635 & 7430 & 23.90 & 413 & 2006 Jan & 0.34 & 0.01 \\
\hline$I R A C 2$ & Spitzer & 45110 & 10110 & 23.30 & 413 & $2006 \mathrm{Jan}$ & 0.33 & 0.01 \\
\hline IRAC3 & Spitzer & 57593 & 14060 & 21.30 & 413 & 2006 Jan & 0.33 & 0.01 \\
\hline$I R A C 4$ & Spitzer & 79594 & 28760 & 21.00 & 413 & 2006 Jan & 0.32 & 0.01 \\
\hline MI PS 24 & Spitzer & 236741 & 50560 & $80 \mu J y$ & 385 & 2006 Jan-2008 Jan & & \\
\hline MI PS70 & Spitzer & 714329 & 184844 & 4 mJy & 34 & 2006 Jan-2008 Jan & & \\
\hline MI PS 160 & Spitzer & 1558938 & 344982 & $30 \mathrm{mJy}$ & 8 & 2006 Jan-2008 Jan & & \\
\hline$L$ & VLA & $1.4 \mathrm{GHz}$ & $75 \mathrm{MHz}$ & $45 \mu J y$ & $139^{\mathrm{h}}$ & 2003 Aug-2006 Mar & & \\
\hline
\end{tabular}

Notes.

${ }^{\text {a }}$ Effective wavelength: $\lambda_{\text {eff }}=\int R * \lambda d \lambda / \int R d \lambda$, where $R$ is the transmission profile normalized to a peak throughput of unity, and including the transmission of the atmosphere, the telescope, the camera optics, the filter, and the detector.

$\mathrm{b}$ The FWHM of the response function.

${ }^{c} 5 \sigma$ in a $3^{\prime \prime}$ aperture for data from infrared to UV bands. Flux limit for the radio and X-ray (in erg $\mathrm{cm}^{-2} \mathrm{~s}^{-1}$ ) data.

${ }^{\mathrm{d}} k_{\lambda}$ is the constant parameter for each band to calculate the Galactic extinction by $A_{\lambda}=k_{\lambda} E(B-V)$.

${ }^{\mathrm{e}}$ Galactic extinction calculated by $k_{\lambda}$ in each band multiplied by mean $E(B-V) 0.0192$ (see Section 4.1.1).

${ }^{\mathrm{f}}$ The rest of the sources are so bright that they saturate in the band.

$\mathrm{g}$ The rest are out of the region of the Hubble coverage.

h 61 sources have $>5 \sigma$ detections, 78 sources have $3 \sigma-5 \sigma$ detections. In addition, 268 other sources have $3 \sigma$ upper limits.

* These photometry data were excluded after variability correction.

** The unit is erg $\left(\mathrm{cm}^{2} \mathrm{~s}\right)^{-1}$.

\subsection{Luminosity and Redshift}

In Figures 2, 3, and 4, the properties of the XC413 sample (black crosses) are compared to the E94 quasars (magenta squares).

Figure 2 shows the rest-frame $2 \mathrm{keV} \mathrm{X}$-ray luminosity versus redshift plane. For the XC413 quasars (black crosses), the $2 \mathrm{keV}$ luminosity is calculated from the observed X-ray band flux and the spectral index $\Gamma$ (Mainieri et al. 2007). For the XC413 quasars without $\Gamma$ estimation, spectral index $\Gamma=2$ for the $0.5-2 \mathrm{keV}$ band and $\Gamma=1.7$ for the $2-10 \mathrm{keV}$ band is assumed (Cappelluti et al. 2009). The magenta squares represent the 47 E94 quasars in the same plot. The $1 \mathrm{keV}$ monochromatic luminosity and the spectral index $\alpha_{x}$ for each E94 quasar 
Table 2

Spectral Line Rest-frame Equivalent Width from SDSS DR7

\begin{tabular}{|c|c|c|c|c|c|c|}
\hline $\begin{array}{l}\text { Spectral } \\
\text { Line }\end{array}$ & $\mathrm{N}^{\mathrm{a}}$ & $\begin{array}{c}\text { Mean }{ }^{\mathrm{b}} \\
(\text { Gaussian}) \\
\text { EW }(\AA)\end{array}$ & $\begin{array}{c}\text { Mean }^{\mathrm{c}} \\
(\text { Log-normal) } \\
\text { EW }(\AA)\end{array}$ & $\begin{array}{c}\sigma^{\mathrm{c}} \\
(\log -\text { normal }) \\
\text { EW }(\AA)\end{array}$ & $\begin{array}{c}\text { Correction }^{\mathrm{d}} \\
r \\
(\mathrm{dex})\end{array}$ & $\begin{array}{c}\text { Correction }^{\mathrm{e}} \\
\text { I A624 } \\
(\mathrm{dex})\end{array}$ \\
\hline Ly $\alpha$ & 4 & 55.3 & 58.6 & 50.5 & 0.052 & 0.200 \\
\hline CIV & 39 & 26.3 & 26.7 & 15.2 & 0.024 & 0.103 \\
\hline $\left.\mathrm{C}_{\text {III }}\right]$ & 70 & 17.6 & 17.6 & 6.7 & 0.016 & 0.070 \\
\hline $\mathrm{Mg}_{\text {II }}$ & 137 & 20.8 & 20.7 & 8.4 & 0.019 & 0.082 \\
\hline $\mathrm{H} \gamma$ & 21 & 13.4 & 13.5 & 6.1 & 0.013 & 0.055 \\
\hline $\mathrm{H} \beta$ & 25 & 29.3 & 29.8 & 17.4 & 0.027 & 0.113 \\
\hline [O III $] 4960$ & 19 & 13.7 & 12.4 & 10.4 & 0.012 & 0.051 \\
\hline [O III] 5008 & 19 & 25.0 & 22.3 & 22.5 & 0.021 & 0.087 \\
\hline $\mathrm{H} \alpha$ & 13 & 204.3 & 186.7 & 226.0 & 0.148 & 0.457 \\
\hline
\end{tabular}

Notes.

a This column is the number of detections in our optical (Magellan/MMT/SDSS) spectra for each spectral line.

${ }^{\mathrm{b}}$ Mean EW correspond to the Gaussian fit of the histogram in Figure 12.

${ }^{c}$ The mean and sigma of the log-normal fit of the histogram in Figure 12.

d Typical correction for a broad band for quasars at redshift $z=2$, here we use $r$ band (BW $=1382 \AA$ ) as an example.

The correction is calculated as $\log \left(\mathrm{BW} /\left(\mathrm{BW}+\mathrm{EW}_{\text {rest }} *(1+z)\right)\right)$.

e Typical correction for an intermediate band for quasars at redshift $z=2$, here we use IA624 $(\mathrm{BW}=300 \AA)$ as an example. The correction is calculated as $\log \left(\mathrm{BW} /\left(\mathrm{BW}+\mathrm{EW}_{\text {rest }} *(1+z)\right)\right)$.

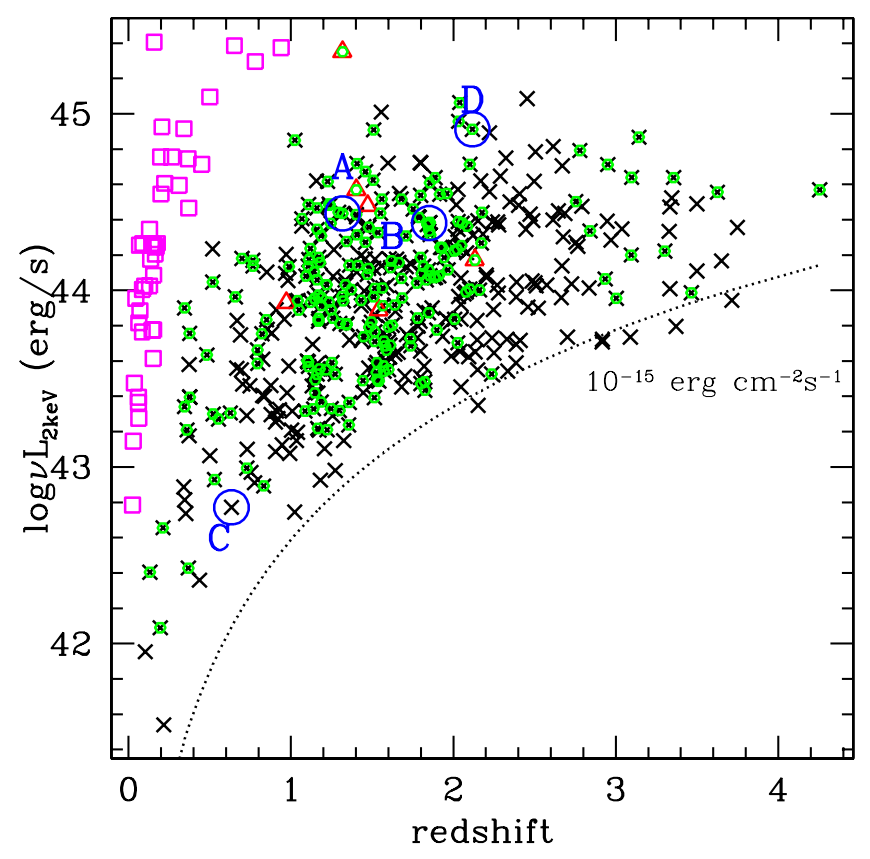

Figure 2. X-ray luminosity vs. redshift for the 413 type 1 AGNs in $X M M$ COSMOS. The depth of the survey is $\sim 5 \times 10^{-16} \mathrm{erg} \mathrm{cm}^{-2} \mathrm{~s}^{-1}(0.5-2 \mathrm{keV})$ and $\sim 3 \times 10^{-15} \mathrm{erg} \mathrm{cm}^{-2} \mathrm{~s}^{-1}(2-10 \mathrm{keV})$ over $90 \%$ of the area. The dotted line shows the X-ray flux of $10^{-15} \mathrm{erg} \mathrm{cm}^{-2} \mathrm{~s}^{-1}$ as a guide of the flux limit. Quasars from E94 are shown as magenta squares. Black crosses = radio-quiet sources, red triangles $=$ radio-loud sources (see Section 3.3 for the definition). Green hexagons $=$ sources corrected for host galaxy contribution (see Section 4.4). The four sources labeled (A, B, C, and D) in blue are discussed in Section 4.5. (A color version of this figure is available in the online journal.)

respectively (E94, Table 2) has been used to calculate the $2 \mathrm{keV}$ monochromatic luminosity of the E94 sample. Note that E94 uses $H_{0}=50 \mathrm{~km} \mathrm{~s}^{-1} \mathrm{Mpc}^{-1}$, so we computed the X-ray luminosity with the same cosmology used in this paper.

In Figure 3, we show the $i$-band absolute magnitude $M_{i}$-redshift plane. For E94 we derived $i$-band magnitudes using the $V$-band magnitude and $(B-V),(R-I)$ colors from E94 (Table 4A), and the transformation from the Johnson system to the AB magnitude in $i$ band given by Jester et al. (2005, Table 1;

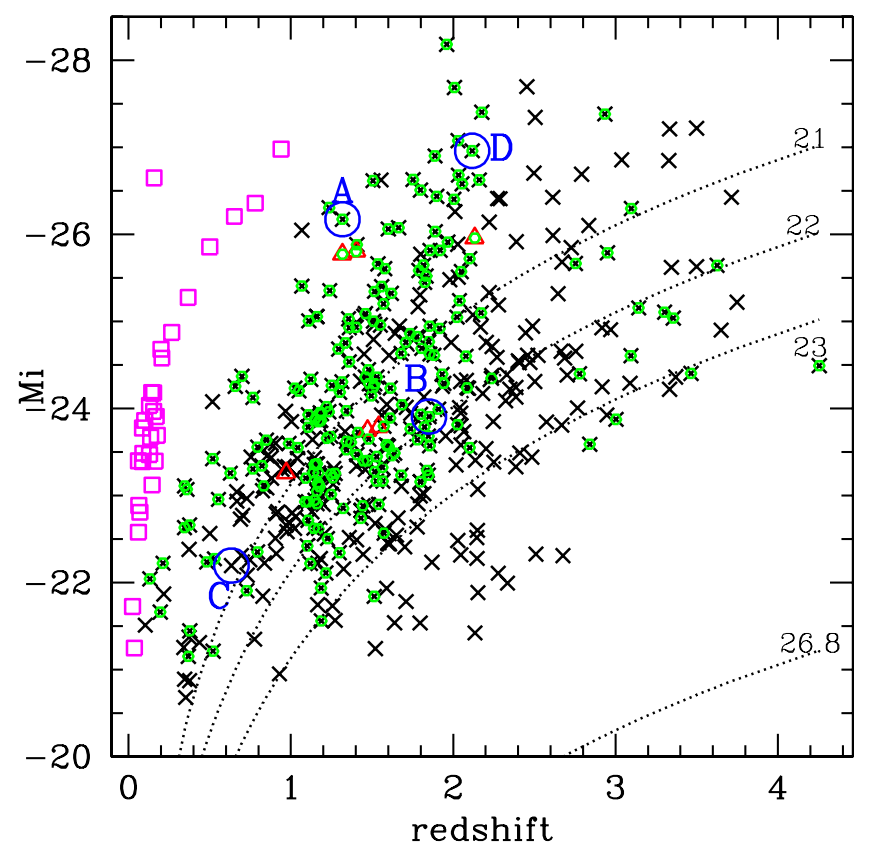

Figure 3. $I$-band absolute magnitude vs. redshift for the 413 type 1 AGNs in $X M M$-COSMOS. The dotted lines represent the SDSS photometry flux limit ( $i=21 \mathrm{mag}$ ), the Magellan and MMT flux limit $(i=23 \mathrm{mag})$, and the photometry flux limit $(r=26.8 \mathrm{mag})$. The symbols are as in Figure 2.

(A color version of this figure is available in the online journal.)

$r-i=0.90(R-I)-0.20$ and $r=V-0.19(B-V)-0.02)$. Note that we first recalculated the tabulated E94 $M_{V}$ with the same cosmology used in this paper. In Figure 4, we show the location of the XC413 sample with respect to the E94 sample in the $i$-band absolute magnitude $M_{i}$ versus X-ray luminosity plane. We can clearly see that the XC413 sample covers a larger luminosity and redshift range compared to the E94 sample.

The XC413 sample is 10 times larger than the E94 quasar sample. Over two orders of magnitude range in luminosity, the XC413 sample homogeneously covers a redshift range (80\% quasars at $0.1 \leqslant z \lesssim 2.2) \sim 6$ times larger than the E94 sample $(80 \%$ quasars at $z<0.35)$, and a flux range some 


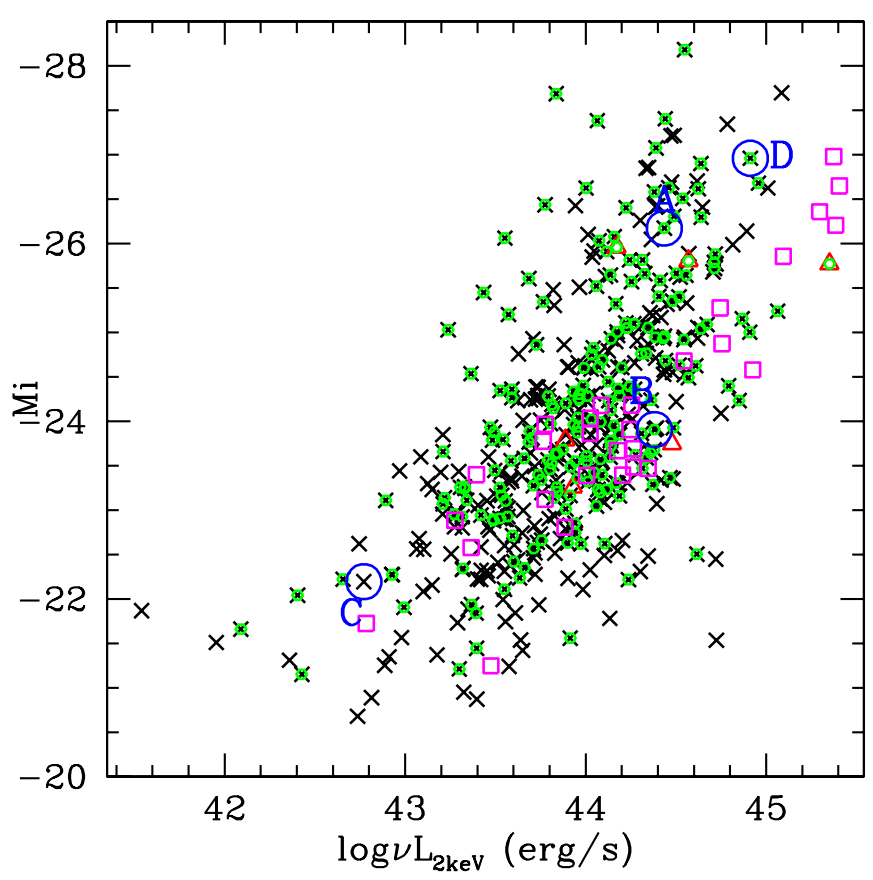

Figure 4. $I$-band absolute magnitude vs. X-ray luminosity for the 413 type 1 AGNs in XMM-COSMOS. The symbols are as in Figure 2.

(A color version of this figure is available in the online journal.)

2 dex fainter than the E94 sample $\left(16.9 \leqslant i_{\mathrm{AB}} \leqslant 24.8\right)$. While the two samples span comparable range of $M_{i}$, the COSMOS sample includes many more lower luminosity $\left(\log v L_{2 \mathrm{keV}}<\right.$ $10^{43.5} \mathrm{erg} \mathrm{s}^{-1}$ ) X-ray sources compared to the E94 sample (see Figure 2).

\subsection{X-Ray Properties}

The hardness ratios of the XC413 AGN are shown versus redshift in Figure 5. The hardness ratio is defined as $\mathrm{HR}=$ $(H-S) /(H+S)$, where $H$ are the counts in the $2-10 \mathrm{keV}$ band and $S$ those in the $0.5-2 \mathrm{keV}$ soft energy band (Brusa et al. 2010). Usually, a negative HR indicates the absence of $\mathrm{X}$-ray absorption, although for a given spectrum the HR is a strong function of redshift. There are 73 (17.6\%) quasars with $\mathrm{HR}=-1$, which is reasonable fraction for type 1 AGNs (e.g., Rosati et al. 2002). There are also six quasars with $H R=1$ in XC413, which is somewhat unexpected. We will discuss the properties of these six quasars in a following paper. Figure 5 also shows the curves of hardness ratio versus redshift for a range of equivalent hydrogen column densities $\left(N_{\mathrm{H}}\right)$, computed using XSPEC (version 11; Arnaud 1996), assuming a spectral slope of $\Gamma=1.7$ and solar abundances. Most (299) of the XC413 AGNs have HR values indicative of small absorbing hydrogen column densities, $N_{\mathrm{H}}<10^{22} \mathrm{~cm}^{-2}$, as is typical of most type 1 AGNs (e.g., Mainieri et al. 2007). However, $27.6 \%$ (114) of the XC413 sample shows HR indicative of obscuration $N_{\mathrm{H}}>10^{22} \mathrm{~cm}^{-2}$ and even larger. Mainieri et al. (2007) estimated the $N_{\mathrm{H}}$ value for 378 quasars of the XC413 from the X-ray spectrum. Only 36 out of the 378 quasars have $N_{\mathrm{H}}$ value larger than $10^{22} \mathrm{~cm}^{-2}$. For the 114 quasars lying above the $N_{\mathrm{H}}=10^{22} \mathrm{~cm}^{-2}$ curve in the HR versus redshift plane, 19 quasars have estimated $N_{\mathrm{H}}>10^{22} \mathrm{~cm}^{-2}, 76$ quasars have estimated $N_{\mathrm{H}}<10^{22} \mathrm{~cm}^{-2}$, and 19 quasars do not have the $N_{\mathrm{H}}$ estimation from the X-ray spectrum. From Figure 5, we can also see that majority of the sources above the $N_{\mathrm{H}}=10^{22} \mathrm{~cm}^{-2}$ curve cluster near the curve within the range of the typical HR error bar. Similar results have

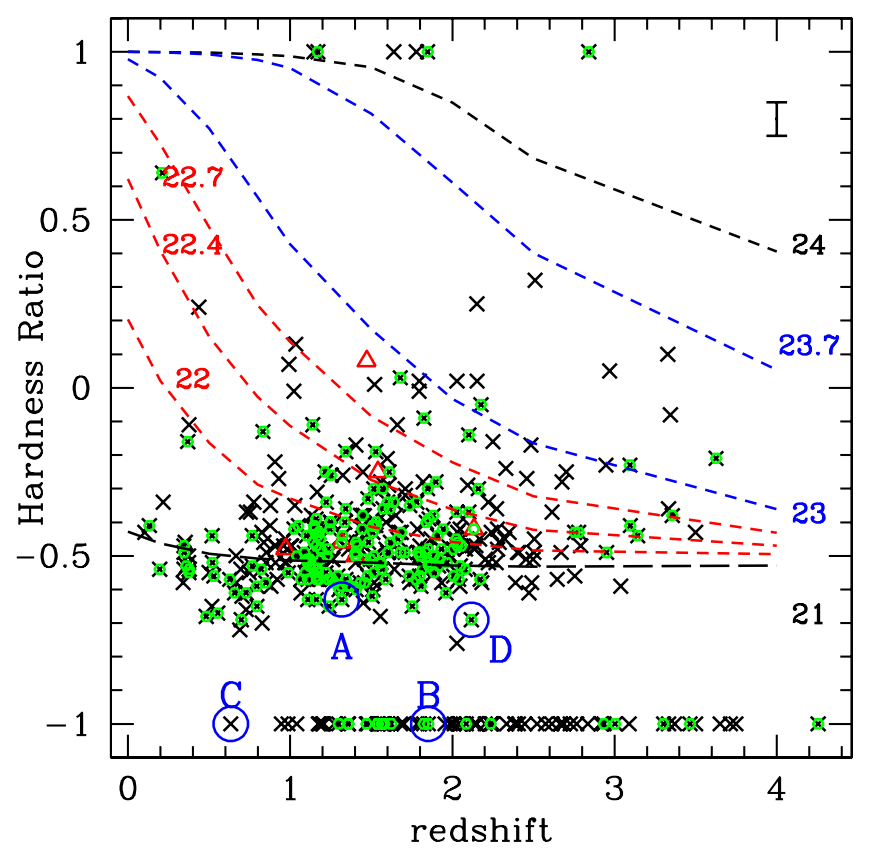

Figure 5. Hardness ratio $((H-S) /(H+S))$ vs. redshift for the XC413 sample. The symbols are the same as in Figure 2. The lines show the tracks for different column density labeled in $\log \left(N_{\mathrm{H}}\right)$. The line in the upper right corner showed the size of the mean error bar of HR.

(A color version of this figure is available in the online journal.)

been found by G. Lanzuisi et al. (in preparation) for the bright sources in the C-COSMOS survey (Elvis et al. 2009).

A deeper analysis of the X-ray properties of this sample, including the optical to X-ray slope analysis, has been reported in Lusso et al. (2010) and Brusa et al. (2010).

\subsection{Radio-loudness}

Quasars are often classified into radio-loud and radio-quiet, based on their radio properties. Typically, $10 \%$ of all quasars are radio-loud (e.g., Kellermann et al. 1989; Urry \& Padovani 1995; Ivezić et al. 2002).

There are several ways to classify quasars as radio-loud or radio-quiet. We tried the following: (1) $R_{L}=\log \left(f_{5 \mathrm{GHz}} / f_{B}\right)$ in the rest frame (Wilkes \& Elvis 1987; Kellermann et al. 1989), for which $R_{L}>1$ defines a radio-loud source; (2) $q_{24}=\log \left(f_{24 \mu \mathrm{m}} / f_{1.4 \mathrm{GHz}}\right)$ in the rest frame and observed frame (Appleton et al. 2004), for which $q_{24}<0$ is defined as radio-loud; (3) $R_{1.4}=\log \left(f_{1.4 \mathrm{GHz}} / f_{K}\right)$ in the observed frame, for which $R_{1.4}>1$ defines a radio-loud source; (4) $R_{u v}=\log \left(f_{5 \mathrm{GHz}} / f_{2500 \AA}\right)$ in the rest frame (e.g., Stocke et al. 1992; Jiang et al. 2007), for which $R_{u v}>1$ defines a radioloud source; (5) $\log _{10}\left[P_{5 \mathrm{GHz}}(\mathrm{W} / \mathrm{Hz} / \mathrm{Sr})\right]>24$ in the rest frame (Goldschmidt et al. 1999) as radio-loud; (6) $R_{X}=$ $\log \left(v L_{v}(5 \mathrm{GHz}) / L_{X}\right)>-3$ in the rest frame (Terashima \& Wilson 2003) as radio-loud.

After cross-checking with all the above criteria, there are six sources classified as radio-loud in all cases. ${ }^{32}$ We define these 6 of the 413 sources as radio-loud sources. For the most commonly used criteria, using the $R_{L}$ criterion, there are 17 radio-loud quasars. Using the $q_{24}$ criterion, there are 13 radio-loud quasars. Using both $R_{L}$ and $q_{24}$ criteria gives eight radio-loud sources. ${ }^{33}$ The fraction of radio-loud sources in the XC413 sample is no

\footnotetext{
32 XID $=40,2282,5230,5275,5517,5395$.

33 The two additional sources using $R_{L}$ and $q_{24}$ criteria are XID $=5497$, 54541 .
} 


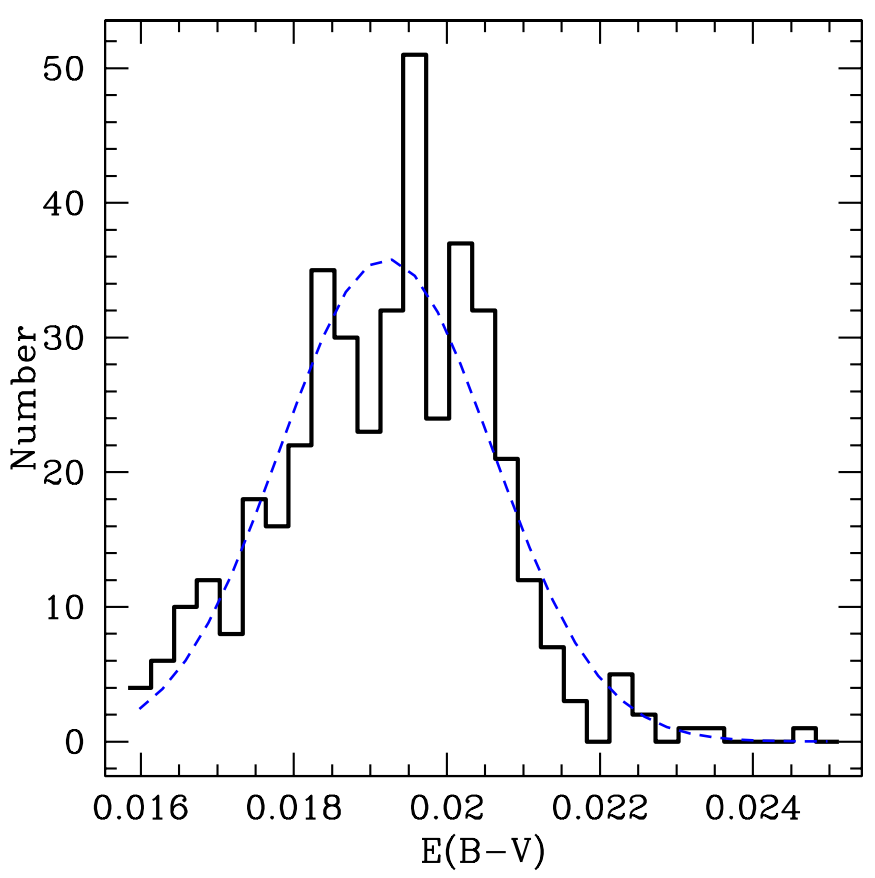

Figure 6. Distribution of estimated Galactic extinction for the XC413 AGN. Blue dashed line = Gaussian fit.

(A color version of this figure is available in the online journal.)

more than $4.5 \%$ using any one criterion, and no more than $2.1 \%$ using two or more criteria, smaller than the $\sim 10 \%$ seen in typical optically selected AGN samples (e.g., Peterson 1997). The Very Large Array (VLA) detection is deep enough that for a great majority of the quasars in XC413 (95.7\%), the VLA data would detect a radio-loud quasar, using any standard criteria. The radio-loudness of the XC413 sample and the effects of the detection limits in radio to this fraction will be discussed in a later paper (Paper IV).

\section{SPECTRAL ENERGY DISTRIBUTIONS}

Our goal is to produce uniform rest-frame SEDs for all the XC413 quasars. To do so, several corrections to the observed SEDs need to be considered. The corrected SEDs can then be converted to a uniform grid in the rest frame to calculate the mean and dispersion.

\subsection{Corrections to the Observed SEDs}

Several complicating factors need to be considered before we can study the SED of the individual sources: (1) Galactic extinction, (2) variability, (3) emission line flux contamination, and (4) host galaxy contamination. In this subsection, we will discuss the first three effects, deferring the host galaxy correction, which is more complex and requires more assumptions, to Section 4.4 .

\subsubsection{Galactic Extinction}

Although the Galactic extinction is small in the COSMOS field, with a median of $E(B-V)=0.0195$ (Capak et al. 2007), we include the correction on a source-by-source basis to eliminate this factor. A photometric correction for each band of the source is calculated from the Galactic extinction of this source multiplied by the filter-dependent factor $k$ given in Table 1. The factors are calculated by integrating the filter

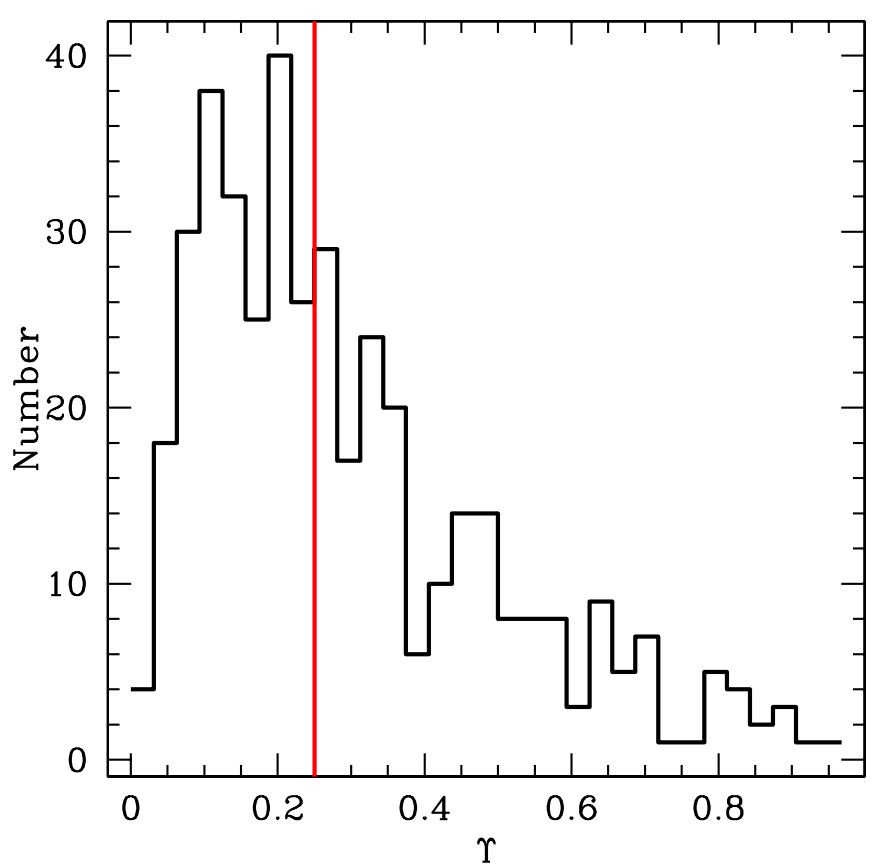

Figure 7. Distribution of the variability parameter $\Upsilon$ (Salvato et al. 2009) of the XC413 AGN. The vertical line divides the sources into variable $(>0.25)$ and not-variable $(<0.25)$.

(A color version of this figure is available in the online journal.)

response function against the Galactic extinction curve from Cardelli et al. (1989).

The estimated $E(B-V)$ from Schlegel et al. (1998) for the $\mathrm{XC} 413 \mathrm{AGN}$ has a mean of 0.0192 and a standard deviation of 0.0014 . For the most affected band, the FUV band, the mean Galactic extinction is $\sim 0.17 \pm 0.01 \mathrm{mag}$, which reduces to $0.10 \pm 0.03$ mag for the shortest wavelength optical band (the CFHT $u$ band). A histogram of $E(B-V)$ for the sample is shown in Figure 6.

\subsubsection{Variability}

The COSMOS optical and infrared data were taken over a 4 year interval, from 2004 to 2007, and the SDSS data for the field were taken as early as 2001 . This long time span causes problems in deriving the true shape of the SEDs, because AGNs are significantly variable on these timescales (Hawkins 2007; Sergeev et al. 2006). Most AGNs vary in their optical continuum flux on the order of $10 \%$ on timescales of months to years (Vanden Berk et al. 2004).

Variability is common in the XC413 sample too. Salvato et al. (2009) analyzed the variability of all the XMM-COSMOS $\mathrm{X}$-ray sample and defined a convenient variability parameter $\Upsilon$ (the rms of the magnitude offsets at the sampled epochs) to quantify the variability of the sources. Salvato et al. (2009) found that $\Upsilon>0.25$ efficiently separates out variable $X M M$-COSMOS sources (including both point-like and extended sources). Half of the XC413 AGNs show significant variability by this criterion (Figure 7).

An example of an AGN SED from this sample (XID = 17), that clearly changes both in flux and in optical/UV slope, is presented in Figure 8(left). The resulting SED is confused when the entire time period is included. This is a widespread problem. Figure 9 shows the broad $\chi^{2}$ distribution obtained in fitting a quadratic function to the SEDs of the full data set. Details of the SED fitting used here will be discussed in Section 4.2.2. 

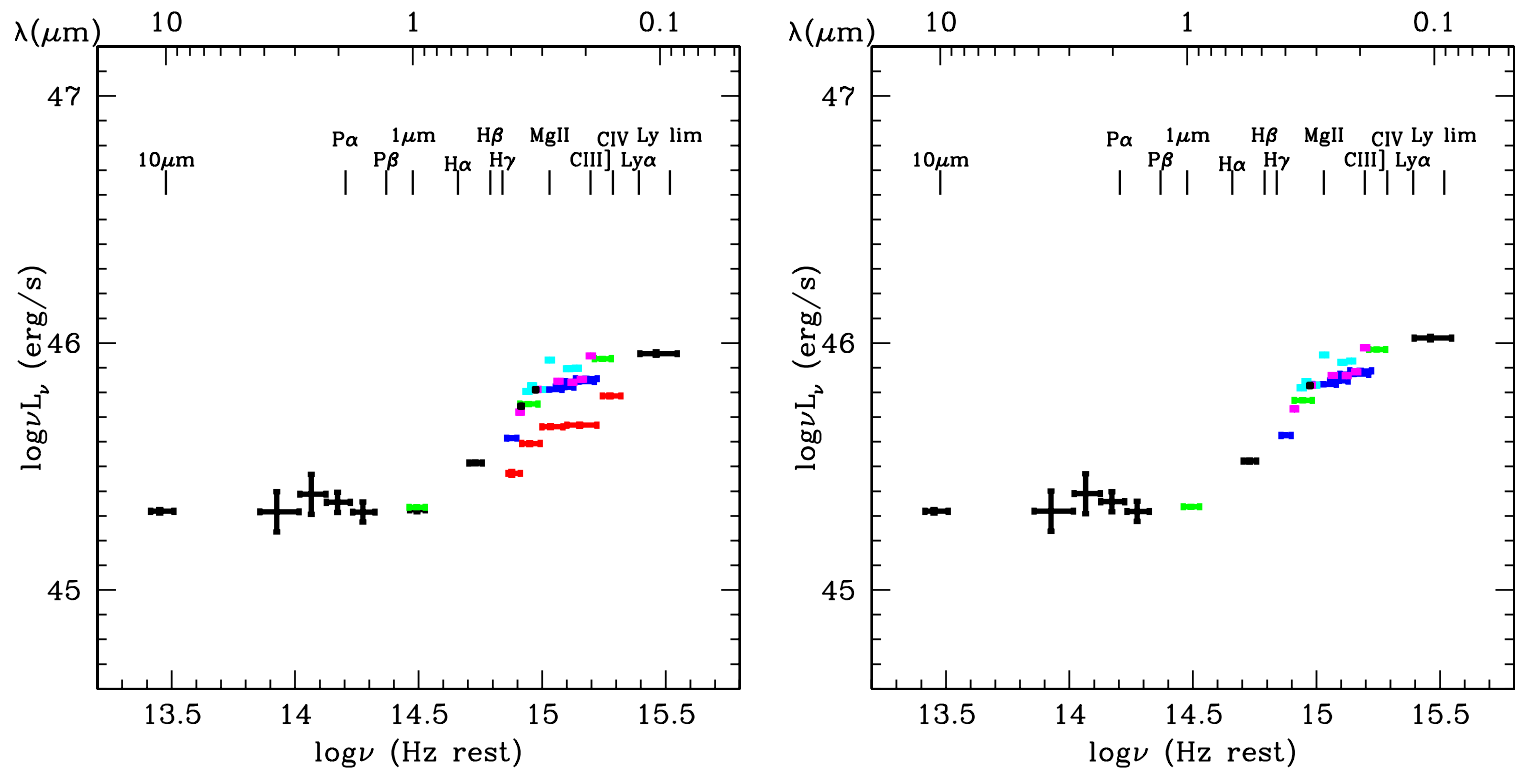

Figure 8. Type 1 AGN SED showing clear flux and slope variability in the optical/UV. The source is COSMOS_J149.85194+1.99845 (XID $=17)$ at $z=1.236$, (i-band absolute magnitude -26.3). Left: SED resulting from using all the data (2001-2007). Right: only the data in the years 2004-2007. From low frequency to high frequency, the black data points are: $24 \mu \mathrm{m}, 8 \mu \mathrm{m}, 5.7 \mu \mathrm{m}, 4.5 \mu \mathrm{m}, 3.6 \mu \mathrm{m}, K$ band, $H$ band, $J$ band, NUV and FUV. The red points show the broad SDSS $u g r i z$ bands from 2001. The blue points are the Subaru broad bands from 2005. The green points are the (CFHT) $K$ band, and the (CFHT) $u$ band and $i$ band. The purple points are the six Subaru intermediate bands for season 1 (2006; IA427, IA464, IA505, IA574, IA709, and IA827). The cyan points are the five Subaru intermediate bands for season 2 (2007; IA484, IA527, IA624, IA679, IA738, and IA767).

(A color version of this figure is available in the online journal.)

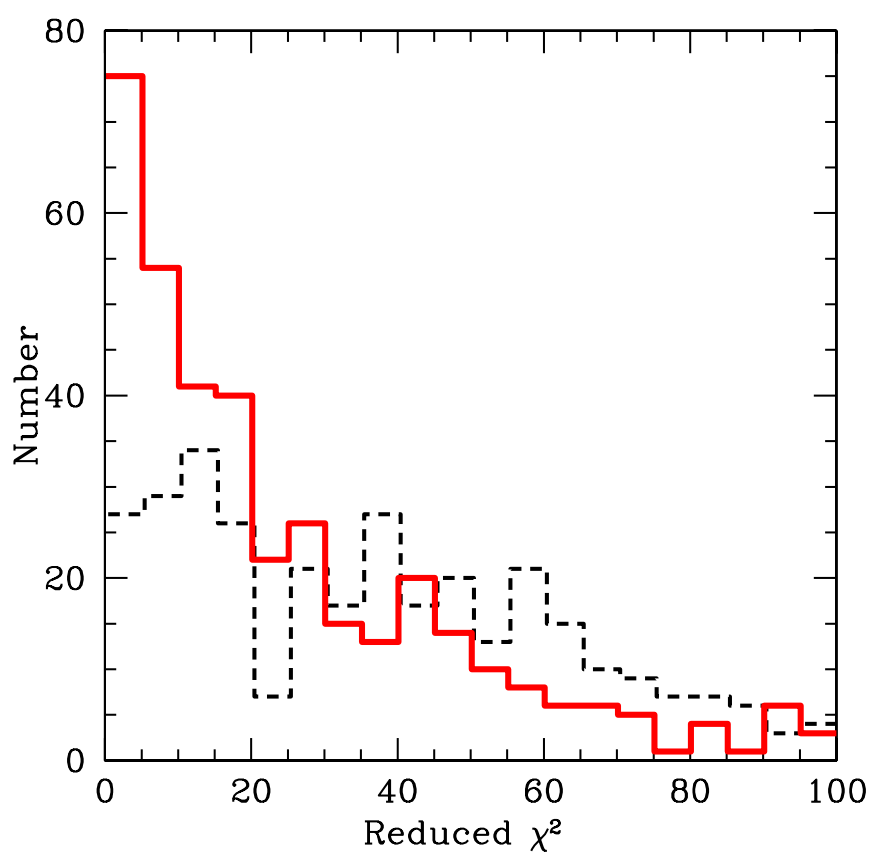

Figure 9. Reduced $\chi^{2}$ of the SED-fitting (see Section 4.2.2) both before (black) and after (red) the restriction of the data to the 2004-2007 interval.

(A color version of this figure is available in the online journal.)

We do not use the Salvato et al. (2009) method to correct the SED, although it works well for deriving photometric redshifts. This is because the method introduces an assumed smooth average SED shape to estimate the correction to be made to each photometry band for variability. This might bias estimates of the intrinsic SED shape.

The alternative approach which we adopt is simply to restrict the data set to a shorter time period. For this approach, we experimented with several different temporal cuts, trying to find the best compromise between contemporaneity and completeness of coverage. Using $\chi^{2}$ fits to the continuum, we find that by using only the data in the interval from 2004 to 2007 reduces the variability issue. Beyond that, we exclude the $K$ band from KPNO because it duplicates the $K$ band from CFHT, which is deeper and has better photometry. We also exclude the data in the NB816 filter because the photometry in this band is the sum of runs distant in time and is thus not reliable due to the variability of the quasars.

Figure 8(bottom) shows how using only the 2004-2007 data removes the scatter in the same AGN (XID = 17) shown in Figure 8(top). Similar improvements are common. The solid line in Figure 9 shows how the reduced time span greatly improves $\chi^{2}$ (with a distinct peak at low $\chi^{2}$ ) after applying these restrictions. The peak reduced $\chi^{2}$ is still $\sim 5$, which is mainly due to two reasons: (1) the photometry of the data is so good that deviations from the simple assumed continuum, due e.g., to weak emission lines and blends, as well as weak residual variability, remain significant; (2) when calculating the $\chi^{2}$, we use the formal photometric errors from various catalogs, which are usually somewhat underestimated.

The SEDs of $\sim 5 \%$ of the sources remain scattered, as in the example in Figure 10. While some of the points are strongly affected by emission lines (see the next section), even the unaffected points seem to show $\sim 0.3$ dex changes. This indicates more rapid variability than is seen in the majority of the sample.

The 8 photometric bands excluded are starred in Table 1 . We have 35 photometry bands remaining with high photometry quality. This temporal cut gives a sample free of strong variability without having to make any assumptions for the SED shape, and retaining virtually the same photometric coverage.

\subsubsection{Broad Emission Line Fluxes}

The BELs of quasars clearly affect the photometry in numerous objects. Figure 11 shows an example, in which the broad Ly $\alpha$ 


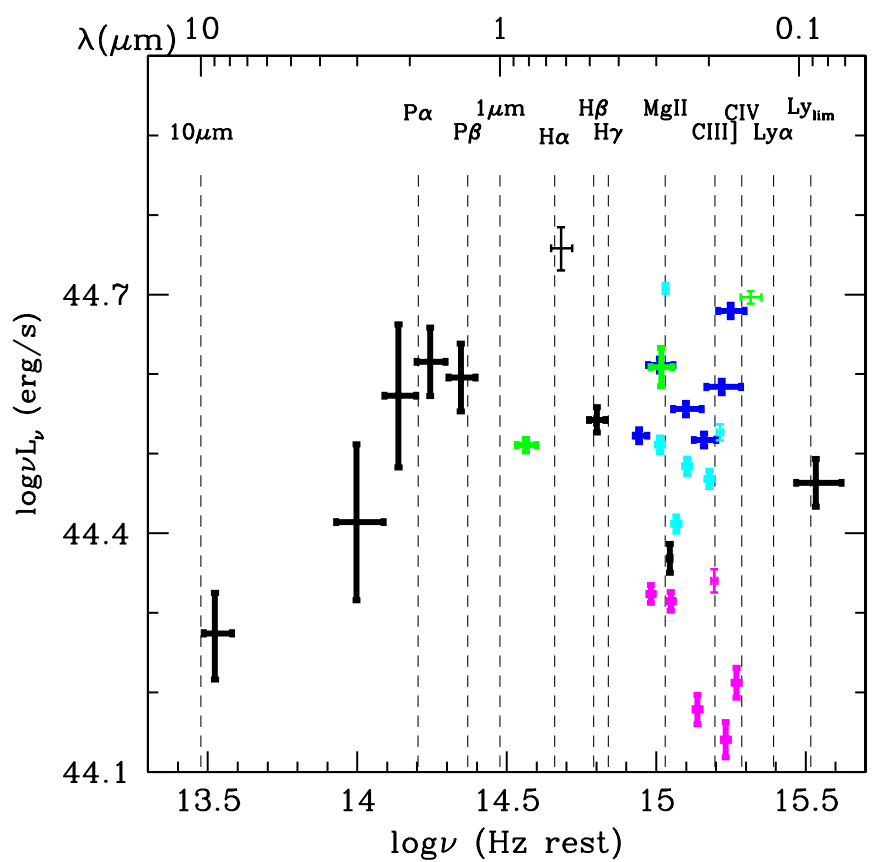

Figure 10. SED (restricted 2004-2007 interval) of source COSMOS_J150.48437+2.16204 (XID = 2232) at $z=1.641$ ( $i$-band absolute magnitude -23.5). The inconsistent photometry, notably in the UV, suggests more rapid variability in this objects, and in $5 \%$ of similar sources, than is typical of the majority of the population. The points are color coded as in Figure 8.

(A color version of this figure is available in the online journal.)

emission line has increased the intermediate band by $\sim 0.3 \mathrm{dex}$ compared to the adjacent broad band. Similarly, the broad C IV emission line has increased the flux by approximately $0.1 \mathrm{dex}$. Visual inspection shows that 280 of the 413 AGNs have photometry that appears clearly affected by BELs.

To correct the SEDs for BEL contamination, we need to measure or estimate equivalent widths (EWs) for all the strong
BELs in the quasar spectra. Although, by construction, we have optical spectra for each of the type 1 AGNs in the sample, these spectra do not span the full spectral range required. Typically, only one or two BELs are present in each optical spectrum. Estimates of EW for most of the BELs are still needed.

For the BELs in the spectra of IMACS/Magellan, VIMOS/VLT, or SDSS (Trump et al. 2007, 2009a; Lilly et al. 2007, 2009; Schneider et al. 2007), the EWs were measured individually using the splot package in IRAF. ${ }^{34}$ The number of measured EWs for the major BELs is shown in the second column of Table 2. The Mg II BEL has measured EWs for $33 \%$ of the sample (137/413), but no other BEL has more than $17 \%$ coverage.

For the remaining BELs, we must estimate their EWs by other means. We considered three methods:

1. Use correlations of EW between the different BELs to bootstrap from the observed lines to the rest.

2. Use the known relationships between $\mathrm{C}$ IV EW and luminosity or redshift, i.e., the Baldwin effect (Baldwin 1977), or analogs for other lines (Green et al. 2001).

3. Take a mean or median EW for each BEL from a survey covering a wide redshift range, in order to cover all the major BELs, and use the dispersion of the observed EW as an error bar on the estimate.

(a) BEL correlations. This method leads to large uncertainties on the BEL EW estimates. We studied the correlation between the EWs of the main BELs using the complete, UV-excess-selected, Palomar-Green X-ray sample with redshift $z<0.4$ (Shang et al. 2007). We found that some line pairs had well-correlated EWs: Mg II and CIV (correlation coefficient, $R=0.93$ ), Ly $\alpha$ and $\mathrm{CIV}(R=0.86), \mathrm{C} \mathrm{III}]$ and $\mathrm{CIV}(R=0.90)$,

\footnotetext{
34 IRAF is distributed by the National Optical Astronomy Observatory, which is operated by the Association of Universities for Research in Astronomy, Inc., under cooperative agreement with the National Science Foundation.
}
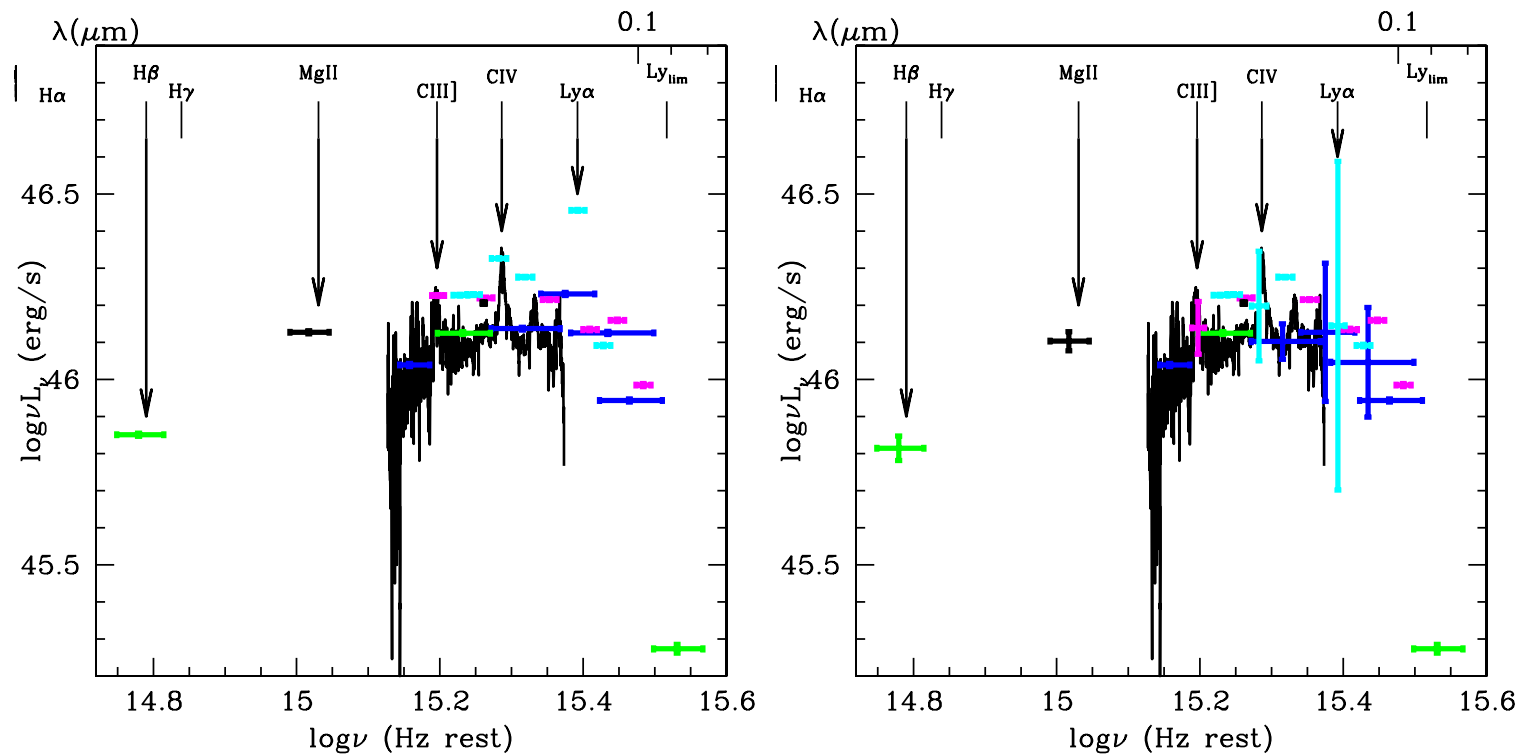

Figure 11. Left: optical SED of COSMOS_J150.20885+2.481935 (XID $=180)$ at $z=3.333$ ( $i$-band absolute magnitude -27.19 ), strongly affected by BELs. Highly significant departures due to $\mathrm{Ly} \alpha$ and $\mathrm{C}$ IV distort fits to the continuum SED by $\sim 0.3$ dex and $\sim 0.1$ dex, and possible BELs of $\mathrm{C}$ III], Mg II, and $\mathrm{H} \beta$ are also apparent. The black solid line show the VLT spectrum of this source. Right: optical SED of COSMOS_J150.20885+2.481935 (XID = 180) after removing the emission lines using the prescription in Section 4.1.3. The error bars on the affected photometry points become larger because of the wide spread in BEL EWs (Figure 12). The black solid line show the VLT spectrum of this source. The points are color coded as in Figure 8.

(A color version of this figure is available in the online journal.) 

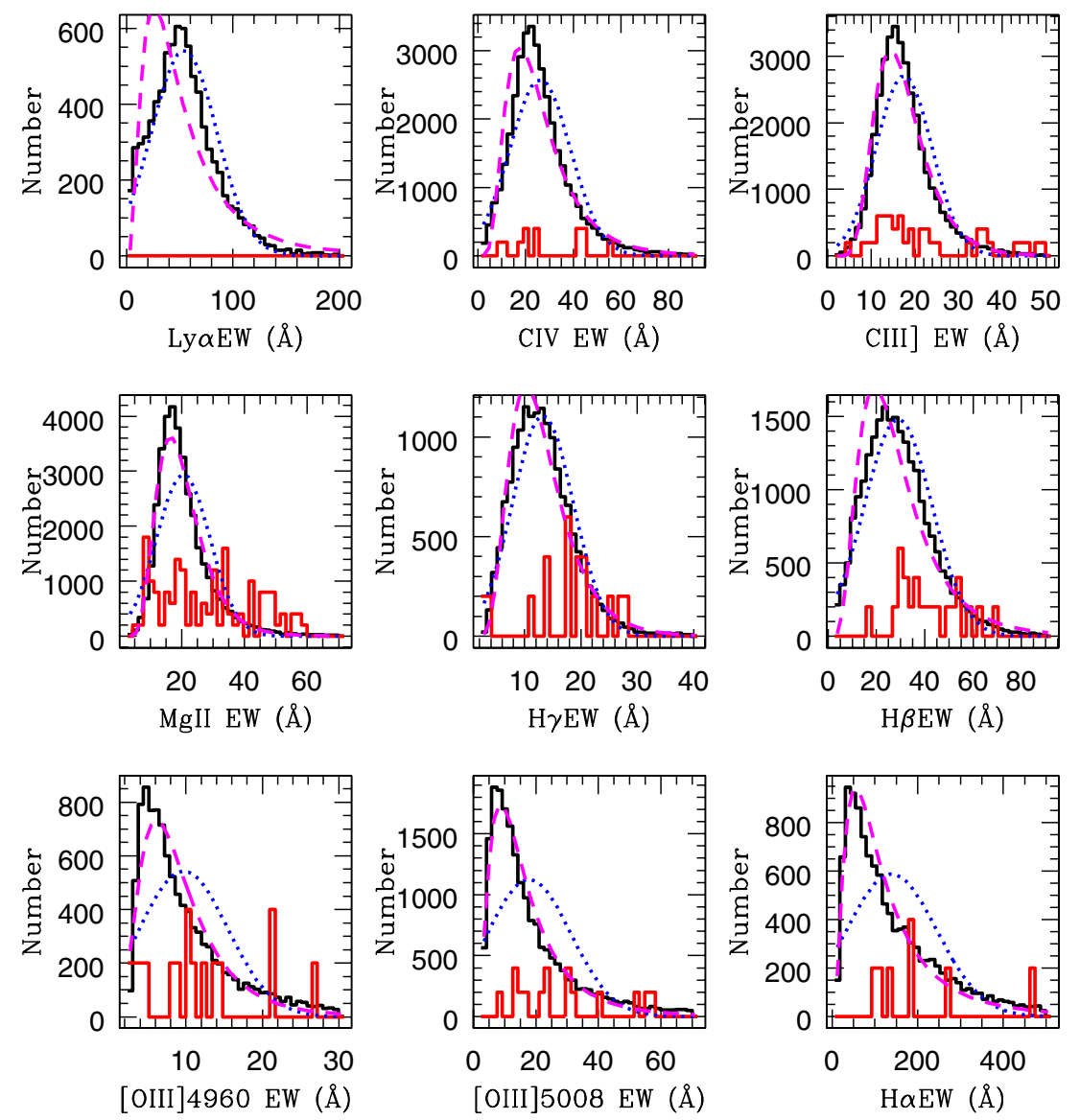

Figure 12. EW distributions of seven BELs and the $[\mathrm{O}$ III] doublet from SDSS DR7 (black histogram). Blue dotted line $=$ Gaussian fit; magenta dashed line $=$ log-normal fit. The EWs measured for the COSMOS type $1 \mathrm{AGN}$ sample is shown as a red solid histogram, multiplied by 200 for plotting purposes. For Ly $\mathrm{y}_{\alpha}$, the four measured EWs are all larger than the range of the histogram.

(A color version of this figure is available in the online journal.)

$\left.\mathrm{C}_{\mathrm{III}}\right]$ and $\mathrm{Mg} \mathrm{II}(R=0.82), \mathrm{H} \alpha$ and $\mathrm{H} \beta(R=0.91)$. However, there was either no or weak correlation between some other key line pairs: the Balmer lines with $\mathrm{Mg}$ II, CIV, CIII], or Ly $\alpha$, and $\operatorname{Ly} \alpha$ with $\mathrm{Mg}$ II $(R<0.5)$. This is quite a surprising result, and may indicate a wider variety of ionizing continua and/or broad-line region conditions and geometry than has been typically considered (Ferland \& Osterbrock 2005). In order to eliminate the effect of different selection methods, we also studied the correlation between BELs for an X-ray-selected survey, the RIXOS survey (Puchnarewicz et al. 1997). We found no strong correlation between any of the BELs $(R<0.4)$ in the RIXOS survey.

(b) The Baldwin effect and its analogs can be studied using EWs from the Large Bright Quasar Survey (LBQS) sample (Green et al. 2001). Green et al. (2001, Figures 1 and 4) show that the widths of the correlations between BEL EW and either monochromatic luminosity at $2500 \AA$ or redshift is $\sim 1$ dex, too large to provide useful EW estimates.

(c) The EW mean and dispersion for each BEL require a large sample to give well-determined values. The obvious choice is the SDSS spectroscopic sample of quasars. We used the EWs of the seven main BELs and the narrow [O III] doublet (Table 2) from SDSS DR7 (Abazajian et al. 2009), as retrieved from the
SDSS archive. ${ }^{35}$ We do not directly use the composite quasar spectrum from SDSS (Vanden Berk et al. 2001), because the EW distribution dispersion for each BEL is not available.

For each quasar, we used only those lines for which the DR7 indicated a good fit $(n \sigma>10)$, which amounts to about $65 \%$ of the SDSS type 1 AGNs. As the cataloged EW are in the observed frame, we transformed them to the rest frame by dividing them by the $(1+z)$, where $z$ is the corresponding redshift of the SDSS quasar. Figure 12 shows the resulting distributions of the restframe equivalent width of the emission lines we consider sorted in wavelength. A Gaussian is a reasonably good approximation for the $\mathrm{Ly} \alpha$, and to a lesser extent, $\mathrm{H} \beta$ and $\mathrm{H} \gamma$ distributions. However, $\mathrm{C}$ IV, $\mathrm{C}$ III], $\mathrm{Mg}$ II, [O III], and $\mathrm{H} \alpha$ show quite skewed distributions, with a tail to large $\mathrm{EW}$, so that a Gaussian is a poor approximation. For these lines we instead fit a log-normal distribution to the histograms. This produced much better fits (Figure 12). The resulting mean and sigma of the log-normal distribution are given in Table 2.

Comparing these three methods, we find that the mean and dispersion of the EW from SDSS provides the best correction.

We adopted the mean and sigma from Table 2 as the mean restframe EW and the error bar in making the BEL subtraction from the SEDs. We made the following simplifying assumption to calculate the BEL correction: when the BEL central wavelength

\footnotetext{
$\overline{35}$ http://www.sdss.org/dr7/products/spectra/index.html
} 
lies within the photometry band, which is equivalent to having more than $1 / 2$ the BEL flux in the filter assuming symmetry, we consider them as if the entire line profile were in the range. When the central BEL wavelength is outside the filter band, we assume the contribution of the BEL is negligible.

We need to transform the rest-frame EW back to the observed $\mathrm{EW}$ of the quasar in XMM-COSMOS sample $\mathrm{EW}_{\mathrm{obs}}=\mathrm{EW}_{\text {rest }} \times$ $(1+z)$. Here, $z$ is the redshift of the quasar undergoing the correction. The EW uncertainties were added to the photometric errors according to the formula below.

Assuming that the continuum flux $f_{c}$ is constant across each broadband photometry interval, then the integrated total flux $\left(v f_{v}\right)$ in the broadband photometry range is $F_{O}$. If the bandwidth is BW, it is easy to see that $F_{O}=f_{c} \times \mathrm{EW}_{\text {obs }}+f_{c} \times \mathrm{BW}$, based on the definition of EW. The integrated continuum flux can then be calculated as

$$
F_{c}=f_{c} \times \mathrm{BW}=\frac{F_{O} * \mathrm{BW}}{\mathrm{EW}_{\mathrm{obs}}+\mathrm{BW}}=\frac{F_{O} \times \mathrm{BW}}{\mathrm{EW}_{\mathrm{rest}} \times(1+z)+\mathrm{BW}} .
$$

So, the correction that needs to be applied to the $\log v f_{v}-\log v$ diagram is $\log \left(\mathrm{BW} /\left(\mathrm{EW}_{\text {obs }}+\mathrm{BW}\right)\right)=\log \left(\mathrm{BW} /\left(\mathrm{EW}_{\text {rest }} \times(1+z)+\right.\right.$ $\mathrm{BW})$ ). The error on the integrated continuum flux is

$$
\begin{aligned}
d\left(\log v f_{v}\right) & =d\left(\log v f_{O}\right)+d\left(\mathrm{EW}_{\mathrm{obs}}\right) /\left(\mathrm{EW}_{\mathrm{obs}}+\mathrm{BW}\right) \\
& =d\left(\log v f_{O}\right)+\frac{d\left(\mathrm{EW}_{\text {rest }}\right)(1+z)}{\mathrm{EW}_{\text {rest }} \times(1+z)+\mathrm{BW}} .
\end{aligned}
$$

As can be seen from the above formula, the correction for the BEL emission is, naturally, larger if the bandwidth is smaller. For broadband photometry, the filter width is of order $\sim 1000 \AA$, while the observed EW of the BELs is of order $\sim 100 \AA$ for the strongest lines, leading to a $\sim 10 \%$ correction to the measurement of the continuum, according to Equation (1). Assuming the error of the measurement of the observed $\mathrm{EW}$ is the square root of the measurement $(\sim 10 \AA)$, the error caused by the correction is then $\sim 1 \%$, according to Equation (2). The Subaru intermediate bands have filter widths of $\sim 200 \AA$ (Table 1), so the correction for these bands is $\sim 3$ times larger. To illustrate how large the correction is, the corresponding corrections for an intermediate band (IA624) and broad band ( $r$ band) are shown in Table 2. Note that no $\mathrm{H} \alpha$ line would lie in these two bands.

As a final step we checked that the correction was reasonable by visual inspection. We find that 217 of the 286 AGNs with clear BEL contributions appear to be well-corrected by the scheme adopted, while 21 appear overcorrected and 48 still appear to have residual BEL emission in their SEDs. The error bars at the positions of the excised BELs are large in the corrected SEDs, due to the additional correction error, which means these affected bands will weigh less in the SED fitting.

Note that previous SED studies (e.g., E94, Richards et al. 2006) do not include the BEL corrections as we did here because the SEDs in those studies were all constructed using broadband photometry, which is affected only at the $1 \%-5 \%$ dex level by the BEL contribution (Table 2, column 6). For $\mathrm{H} \alpha$, which would only be found in the $i$ band and beyond (typically $J$, $H$, or $K$ bands), the effect will be less than $7 \%$ dex. Instead, in COSMOS, the majority of the optical SED comes from the Subaru intermediate bands (seasons 1 and 2, 12 bands), which are affected by the BEL contribution at 5\%-20\% dex level (Table 2, last column). To minimize the confusion to the SED shape from the intermediate bands by this effect, the BEL correction is thus necessary for the XC413 sample.

\subsection{Conversion to a Uniform Rest-frame Grid}

The redshifts of the sample quasars range from 0.1 to 4.3, so any observed photometry point spans a wide range of restframe frequency. To study the individual SEDs, and to calculate a mean SED, we clearly need to shift the SEDs of the quasars to a common rest-frame frequency grid. For ease of comparison to E94, we adopted the grid of the E94 mean SED, which has points separated by 0.02 in logarithmic frequency.

We first converted the flux densities at each frequency for each object to luminosity, and shifted the observed frequencies to the rest frame for each source. We then tried two techniques for interpolating the observed photometry to the uniform grid points: linear interpolation and polynomial fitting. Both methods have advantages and disadvantages. In Section 4.3, we will see that the resulting mean SED using both methods agree well with each other with difference less than 0.02 dex in rest-frame $0.1-10 \mu \mathrm{m}$ (Figure 15). The wavelength/frequency discussed in this section are all in rest frame.

\subsubsection{Linear Interpolation}

The simplest way to produce a uniformly sampled SED is to linearly interpolate between the data points in $\log v L_{v}$ versus $\log v$ space (i.e., connecting the individual points with a power law in linear space).

The COSMOS photometry for the XC413 sample is $>90 \%$ complete from $u$ (CFHT) to MIPS $24 \mu \mathrm{m}$ (Table 1). The apparent drop in the $i$ band from Subaru is due to saturation; the remaining objects are picked up in the $i$ (CFHT) data. The $H$ band (Calar Alto) is only $61 \%$ complete, but the neighboring $J, K$ data is complete making interpolation straightforward. So, over the $1.8 \mathrm{dex}$ wide $0.35 \mu \mathrm{m}-24 \mu \mathrm{m}(\sim 0.14 \mu \mathrm{m}-10 \mu \mathrm{m}$ for the typical $z=1.5$ of XC413) observed frame interpolation is unproblematic.

For the mid-infrared part of the SED the $70 \mu \mathrm{m}$ and especially the $160 \mu \mathrm{m}$ detections become sparse (8\% and $2 \%$, respectively). For the detections we joined the $24 \mu \mathrm{m}$ data to the longer wavelength points with a power law in $\log v f_{v}$ versus $\log v$ space. For non-detections we extrapolated from the rest-frame $24 \mu \mathrm{m}-8 \mu \mathrm{m}$ slope. We checked if the extrapolation generally works by checking the SEDs of the 34 quasars with MIPS70 detection (3 of them also have MIPS160 detection). We found that only for $17.6 \%$ quasars (6 out of 34 ), the extrapolation of the $24 \mu \mathrm{m}$ to $8 \mu \mathrm{m}$ exceed the observations. For half of the six quasars, the deviation is within $1 \sigma$, which means the extrapolation only fails in $8.8 \%$ of the case. So, we use this extrapolation for all the XC413 quasars without $70 \mu \mathrm{m}$ and $160 \mu \mathrm{m}$ detections.

In the sub(millimeter) band from $100 \mathrm{GHz}(3 \mathrm{~mm})$ to $160 \mu \mathrm{m}$ part, the SED can be approximated by the red end of the gray body $f_{v} \propto v^{3+\beta} /\left(e^{h v / k T}-1\right)$, when $h v \ll k T, f_{v} \propto v^{2+\beta}$ (e.g., Lapi et al. 2011). $\beta$ is generally chosen in the range 1-2 (Dunne $\&$ Eales 2001), and we used $\beta=1$. I.e., we assume a power-law $f_{v} \propto v^{3}$ in this band.

For the radio band, for each source with a $>3 \sigma$ detection, we assumed a power-law $f_{v} \propto v^{-0.5}$ (e.g., Ivezić 2004) in the rest-frame $1.4 \mathrm{GHz}(21 \mathrm{~cm})$ to $100 \mathrm{GHz}(3 \mathrm{~mm})$ range. The radio power is never a significant contributor to the total luminosity ( $<3 \%$; Hao et al. 2012a). For sources with only a radio upper limit, we extend the power law of $f_{v} \propto v^{3}$ in the sub(millimeter) to the radio wavelength.

Turning to the high frequency SED, the bluest band in the optical with complete coverage is the $u$ band at $0.38 \mu \mathrm{m}$. In the 

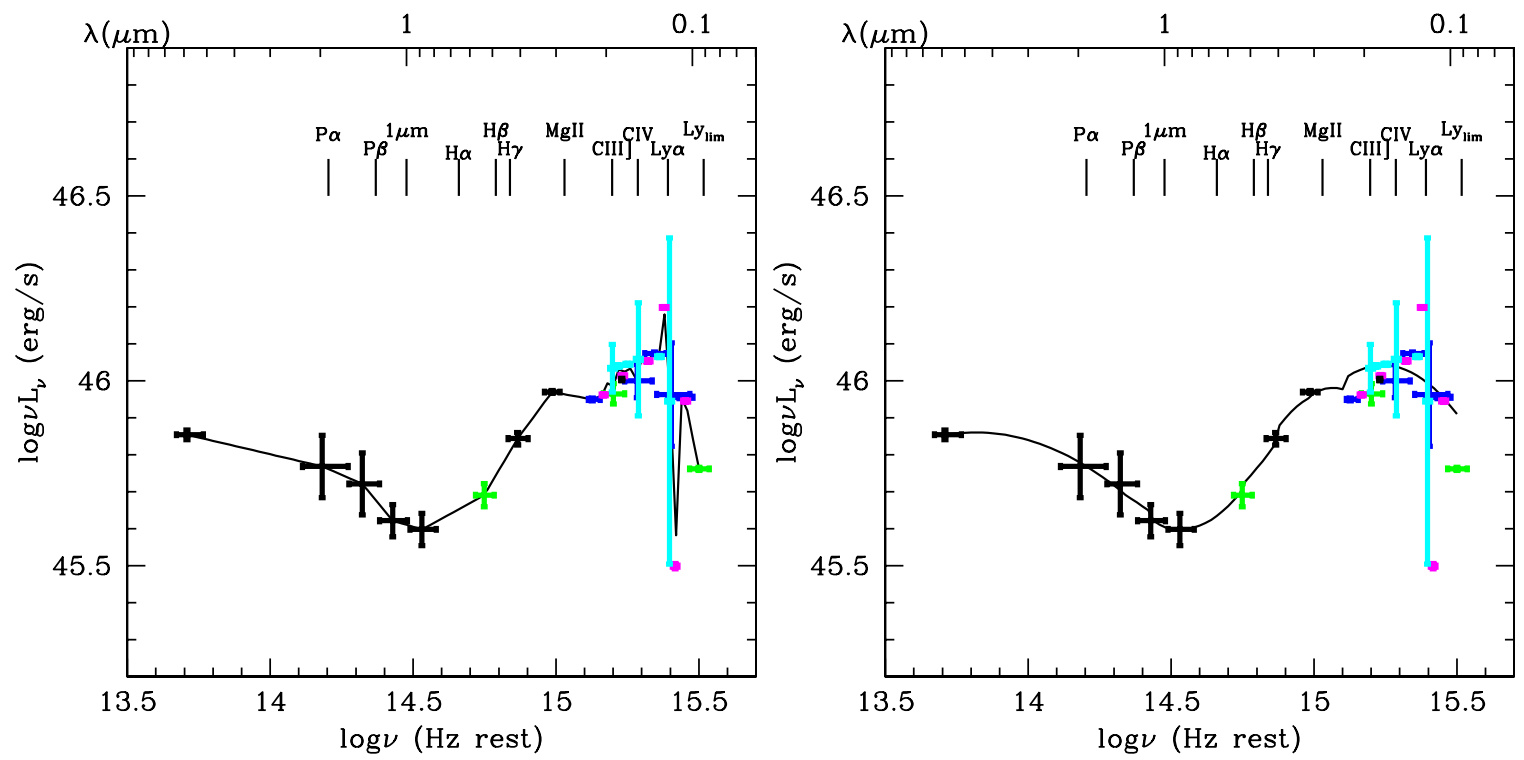

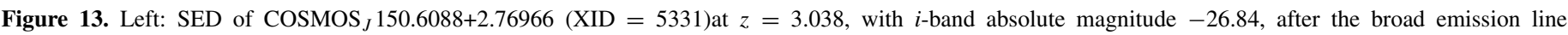

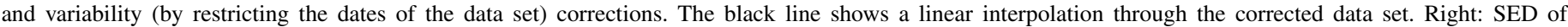

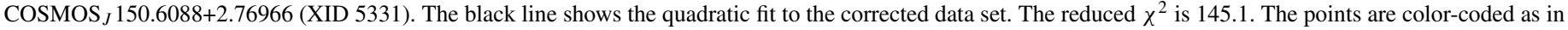
Figure 8.

(A color version of this figure is available in the online journal.)

ultraviolet the COSMOS photometric completeness drops to $60 \%$ at $0.23 \mu \mathrm{m}$ (GALEX NUV) and $33 \%$ at $0.15 \mu \mathrm{m}$ (GALEX FUV). The FUV points would add little to the SED study as FUV fluxes are strongly reduced by the $\operatorname{Ly} \alpha$ forest induced break at $0.12 \mu \mathrm{m}$ for $z \gtrsim 0.3$, i.e., the great majority of the sample $(408 / 413=99 \%)$. The Ly $\alpha$ forest affects the NUV photometry for $z>0.9(358 / 413=87 \%$ of XC413). Almost all $(146 / 150)$ the quasars without NUV detection are at $z>0.9$ and all the quasars without FUV detection are at $z \gtrsim 0.3$.

For the X-ray band, we used the photon spectral index ( $\Gamma)$ for the 318 objects in Mainieri et al. (2007). For the remainder we assumed flat X-ray spectrum $\Gamma=2$ (Cappelluti et al. 2009). The observed $0.5 \mathrm{keV}-10 \mathrm{keV}$ fluxes correspond to a rest-frame band of $1.25 \mathrm{keV}-25 \mathrm{keV}$ at $z=1.5$. We thus extrapolated the observed flux to get the luminosity in the restframe $0.5 \mathrm{keV}-40 \mathrm{keV}$ band using the power-law slope $2-\Gamma$ in the $\log v f_{v}$ versus $\log v$ space.

To join the soft X-rays to the UV through the unobservable EUV band we directly connect the detections in the optical or $\mathrm{UV}$ to the $0.5 \mathrm{keV}$ flux with a power law in $\log v f_{v}$ versus $\log v$ space in keeping with the findings of Laor et al. (1997).

Although linear interpolation is simple, this method could be inappropriate for several reasons:

1. The optical data are rich, and overlapping of bands occurs frequently in this range. The data were taken over a four year time interval, so that fluxes in adjacent and overlapping bands can be discontinuous;

2. The EW correction for the BELs is not perfect, which could drag the 'continuum' to deviate from the correct value;

3. The fluxes in different filters have different error bars and so should be weighted differently, which cannot be done by interpolation.

These factors can lead to poor continuum fits when a linear interpolation is used, and also does not make use of the information contained in the error bars.

\subsubsection{Polynomial Fitting}

An alternative to the linear interpolation is to make a weighted fit of the data using a low order polynomial. We tried several curve fitting methods and found that a simple least squares quadratic fit works well.

For each source, we fit quadratic functions to the observed data from rest-frame $9000 \AA$ to the Lyman limit $(912 \AA)$. The longer wavelength data $(1-160 \mu \mathrm{m})$ were interpolated with a second quadratic function. We smooth the junction of the two quadratics by quadratic interpolation with a smoothing window of 10 grid points, corresponding to a factor of 1.6 in frequency. The disadvantage of this fitting method is that a particular functional form is assumed, which may not be a good representation of the true SED shape of the AGN. Just over $50 \%$ of the sources gave reduced $\chi^{2}<20$, showing that additional structure, or residual variability, is likely to be present. An example of the linear integration and the quadratic fit is shown in Figure 13. For this quasar, the reduced $\chi^{2}=145.1$.

The resulting SEDs for each of the objects in the XC413 are available as VO compatible FITS files in the online version of the journal.

\subsection{Full Sample Mean SED and Dispersion}

The Galactic extinction, variability and emission line corrections done in the previous sections are based on reasonable assumptions without a strong model dependency. We can check the mean and dispersion of the SEDs after these corrections.

We used both the linearly interpolated SEDs and the SED fitting to calculate the mean and dispersion of the sample SEDs. We calculated the arithmetic mean of the $\log v L_{v}$ at each frequency of the grid. As described above, in the rest-frame infrared to ultraviolet range, both methods are based only on detection (see details in Section 4.2.1).

The mean SED of the XC413 AGN, after applying all the above corrections, is shown in Figure 14(left) as a black solid line, as well as the E94 mean radio-quiet and radio-loud SEDs (red and green dashed lines, respectively). For comparison, we 

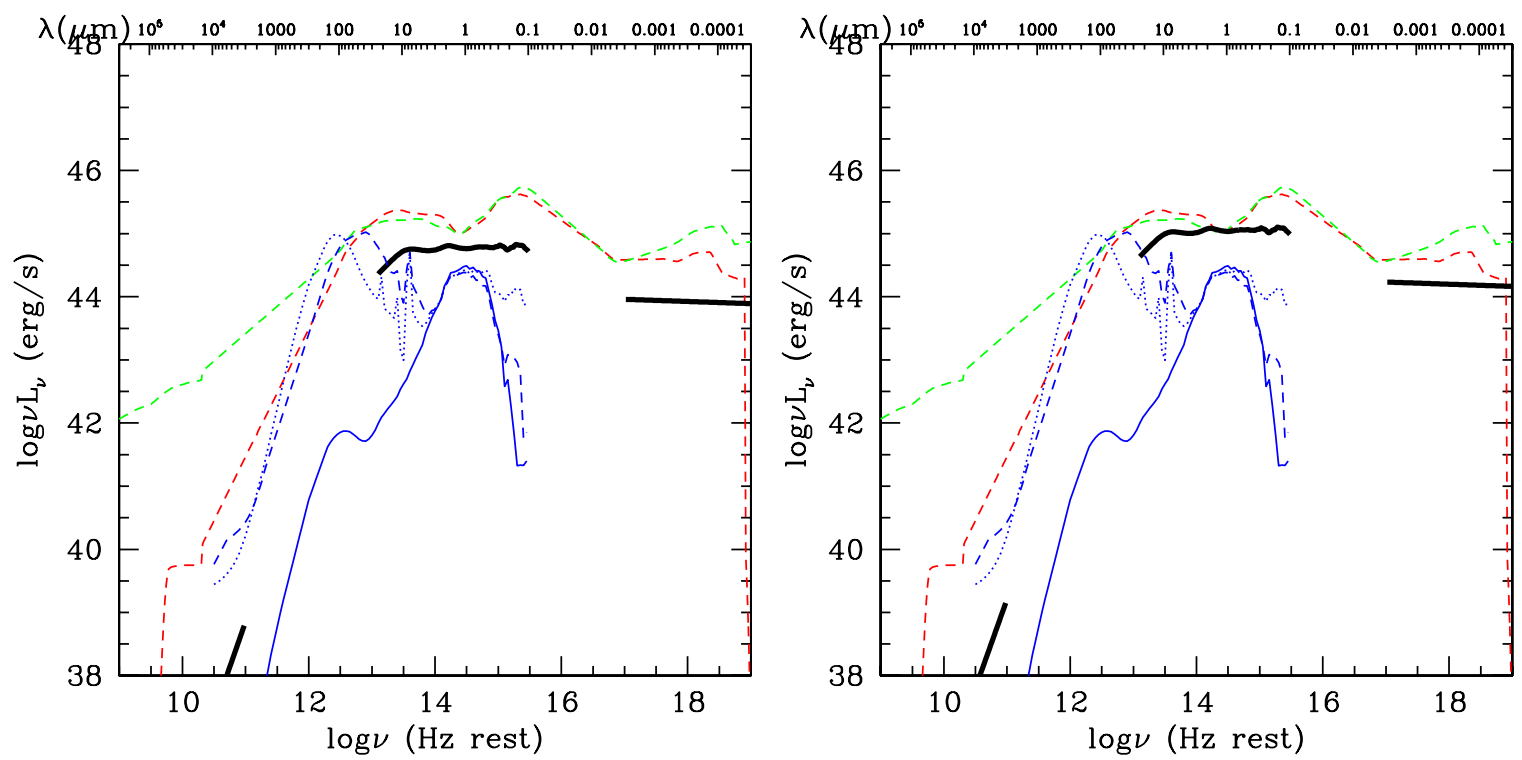

Figure 14. Mean SED for all the quasars in XC413 after the Galactic extinction correction, variability constraints, and broad emission line correction (black solid line): arithmetic mean of the $\log v L_{v}$ (left); arithmetic mean of the $\log v L_{v}$ after normalization at $1 \mu \mathrm{m}$ (right). The dashed lines are the mean radio-loud (green) and radio-quiet (red) SEDs from E94. The blue lines show galaxy templates from Polletta et al. (2007), normalized to the UKIDSS $L_{K}^{*}$ value: blue solid line $=$ elliptical galaxy with 5 Gyr of age (El15); blue dotted line = Spiral galaxy (Spi4); blue dashed line = a starburst galaxy (NGC 6090).

(A color version of this figure is available in the online journal.)

also plot the mean SED normalized at $1 \mu \mathrm{m}$, as for the E94 mean SEDs (Figure 14, right), to avoid a bias toward highluminosity sources. The XC413 mean SED is definitely flatter at all wavelengths than both E94 SEDs.

Three galaxy templates from SWIRE ${ }^{36}$ (Polletta et al. 2007) are also shown in the same plot (Figure 14): a spiral galaxy (Spi4), an elliptical galaxy with an age of $5 \mathrm{Gyr}$, and a starbust galaxy (NGC 6090). These templates are normalized to the value of $\log L_{K}^{*}=44.32\left(M_{K}^{*}=-23\right)$ from the UKIDSS Ultra Deep Survey (Cirasuolo et al. 2007). The XC413 mean SED could be flat because affected by contamination from a strong host galaxy component.

We compare the infrared to ultraviolet mean SED before and after the corrections for Galactic reddening, variability and BEL EW on a much expanded scale in Figure 15. The differences are strongly correlated with the location of the BELs, meaning that that the BEL has been properly corrected in at least eight out of the nine (90\%) lines. Even though the EW distribution (Figure 12) does not show a peculiar behavior, the C IV emission line correction, as derived from SDSS, is not sufficient in our sample $(\sim 0.02$ dex higher than continuum). The polynomial fit makes the SED shape smoother compared to linear interpolation, however the difference between the two methods is less than $0.02 \mathrm{dex}$ in the $0.1-1 \mu \mathrm{m}$ range.

Concentrating on the UV to IR $(0.1-10 \mu \mathrm{m})$ range, it is clear from Figure 14 that the mean SED of the XC413 sample is quite flat, and lacks the clear $1 \mu \mathrm{m}$ inflection point between the UV and near-IR bumps seen in E94. E94 found that host galaxy corrections were significant even in the most luminous quasars in their sample. ${ }^{37}$

\footnotetext{
36 The 16 galaxy templates in the "SWIRE Template Library" (Polletta et al. 2007) include: 3 elliptical galaxy templates "El12," "El15," and "El113"

representing elliptical galaxy of age $2 \mathrm{Gyr}, 5 \mathrm{Gyr}$, and $13 \mathrm{Gyr}$, respectively; 7 spiral galaxy templates "S0," "Sa," "Sb," "Sc," "Sd," "Sdm," and "Spi4"; and 6 starburst galaxy templates "NGC 6090," "M82," "Arp220,"

"IRAS20551-4250," "IRAS22491-1808," and "NGC 6240."

37 In retrospect, this result, like that of McLeod \& Rieke (1994) and Eskridge et al. (1995), was an early hint of the correlation between host bulge mass and central black hole mass (Magorrian et al. 1998).
}
$\lambda(\mu \mathrm{m})$ 1
0.1

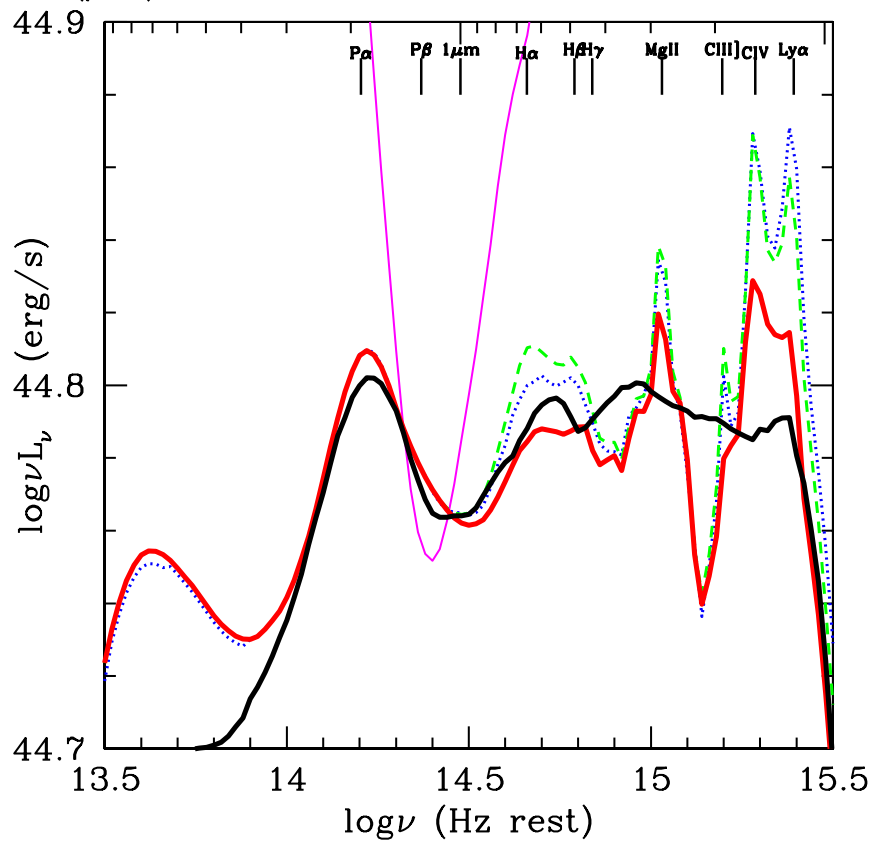

Figure 15. Comparison of the mean IR to UV XC413 SED derived by different methods. (Note that the $y$-axis has a much expanded scale compared with Figure 13). Blue dotted line = "raw" mean SED before corrections; green dashed line = mean SED after the Galactic extinction correction and variability restriction. The two solid lines are the mean SEDs after the Galactic extinction correction, variability restriction and BEL EW correction: red line = mean SED by linear interpolation; black line = mean SED by polynomial fitting. The E94 radio-quiet mean SED is shown in magenta solid line.

(A color version of this figure is available in the online journal.)

The dispersion of the SEDs, computed using the median SED and the 68, 90 and 100 percentile contours on each side of the median, is shown in Figure 16, together with the E94 SED. The upper 90th percentile SED shows an E94 like shape, while the lower 90th percentile SED is close to the galaxy templates 

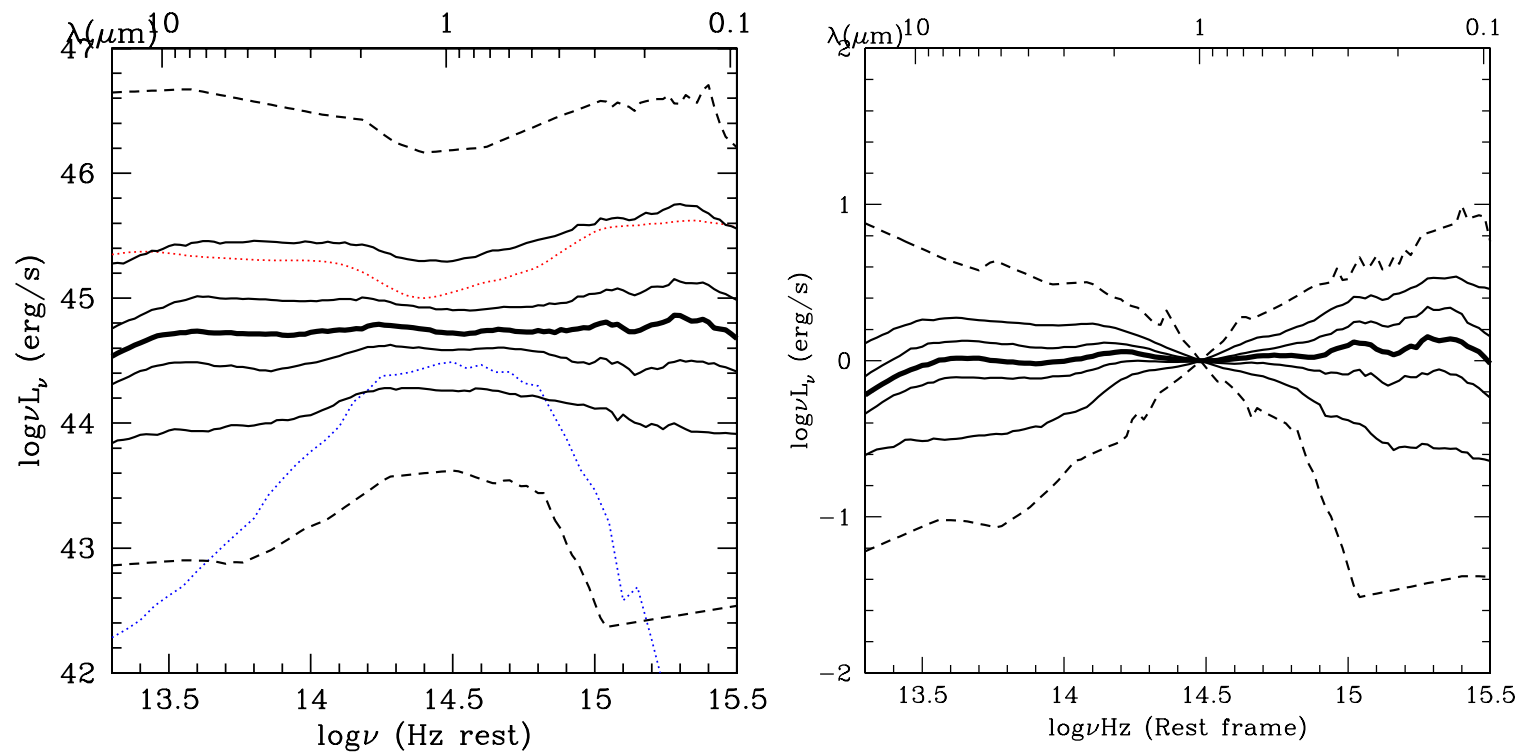

Figure 16. Pre-host subtraction median (thick black line) SED and the 68\%, 90\% (thin solid black lines), and 100\% (dashed black lines) percentile envelopes in the ultraviolet to infrared $(0.09 \mu \mathrm{m}-24 \mu \mathrm{m})$ range: without normalization (left); normalized at $1 \mu \mathrm{m}$ (right). Red dotted line $=$ E94 RQ mean SED. Blue dotted line $=$ 5 Gyr elliptical galaxy template (Ell5).

(A color version of this figure is available in the online journal.)

shown in Figure 16. Typically, X-ray-selected AGN samples, such as XMM-COSMOS, include more sources with low AGN to host galaxy contrast, compared to optically selected samples, and so are more affected by the host galaxy contribution. Due to the bias of the optical color selection, optically selected samples tend to select sources with clear big blue bumps, thus missing the many quasars with large host contributions (compare to Richards et al. 2006 and Luo et al. 2010).

\subsection{Host Galaxy Correction}

K. Jahnke et al. (in preparation) use the COSMOS highresolution Hubble Space Telescope/Advanced Camera for Surveys (HST/ACS) F814W image (Koekemoer et al. 2007) to estimate the host galaxy luminosity and AGN luminosity for $X M M$-COSMOS sources at $z<1$. We could use their results by normalizing a galaxy template to the galaxy luminosity at the observed frequency and subtracting the galaxy contribution from the observed SED. However, this method does not work well for our sample, for the following reasons:

1. We have to assume the host galaxy type in which the AGN is harbored. The F814W band would be at $\sim 3000 \AA$ A rest frame for a typical XC413 quasar at redshift $z=1.5$, which lies on the steeply falling blue side of the galaxy template, so that a small error in template slope (or, effectively, in the age of the youngest population in the host) would lead to a large error in the normalization (Figure 17). In the XMM-COSMOS sample, this issue leads to a severe oversubtraction problem in some cases. For a $5 \mathrm{Gyr}$ old elliptical galaxy template, 9 out of the 89 sources with host magnitude estimation are oversubtracted (10\%). For spirals and star-burst galaxies, this method completely fails, as the oversubtraction fraction is $>80 \%$.

2. Direct imaging works only for the 89 sources at low redshift $(z<1)$ for which the HST F814W image gives a good host galaxy magnitude estimates (K. Jahnke et al., in preparation). For the remaining 324 sources in the sample, we cannot perform a host galaxy correction with this method.

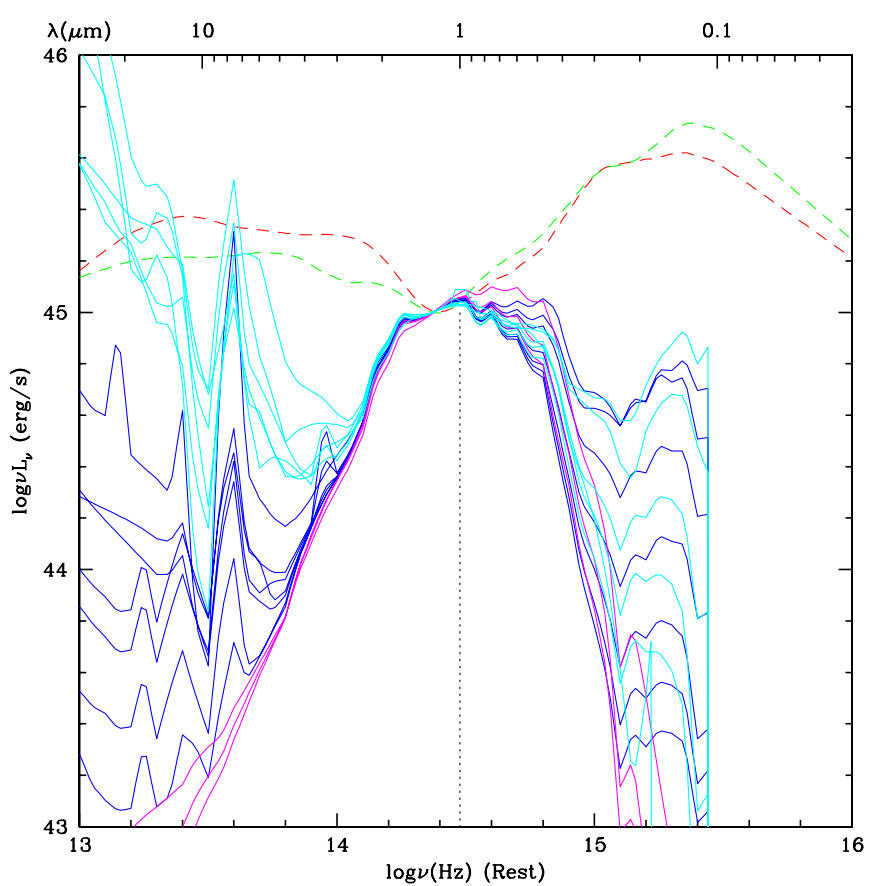

Figure 17. SEDs of 16 galaxy templates from the SWIRE template library (Polletta et al. 2007) normalized at rest-frame $J$ band are shown as solid lines (blue = spiral galaxy: S0, Sa, Sb, Sc, Sd, Sdm, and Spi4; magenta = elliptical galaxy with $2 \mathrm{Gyr}, 5 \mathrm{Gyr}$, and $13 \mathrm{Gyr}$ of age; and cyan = starburst galaxy: NGC 6090, M82, Arp220, IRAS20551-4250, IRAS22491-1808, and NGC 6240). The E94 mean SEDs are shown as dashed lines (red as radio-quiet and green as radio-loud).

(A color version of this figure is available in the online journal.)

Estimates of host luminosity have been made using other techniques:

1. Vanden Berk et al. (2006) use the eigenspectrum decomposition technique to give a host to AGN relationship in the $r$ band, which can be transformed to luminosity as follows 
(Richards et al. 2006, hereinafter R06):

$$
\log \left(L_{r, \mathrm{Gal}}\right)=0.87 \log \left(L_{r, \mathrm{AGN}}\right)+2.887-\log \lambda_{E},
$$

where $\lambda_{E}$ is the Eddington ratio.

2. Marconi \& Hunt (2003) analyze two-dimensional images of nearby galaxies with a black hole mass determined with direct gas kinematics or stellar dynamics (Tremaine et al. 2002) in the local universe. Their results can be transformed as below:

$$
\begin{aligned}
& \log \left(L_{B, \mathrm{Gal}}\right)=0.84 \log \left(L_{\mathrm{bol}}\right)+3.914-0.84 \log \lambda_{E} \\
& \log \left(L_{J, \mathrm{Gal}}\right)=0.88 \log \left(L_{\mathrm{bol}}\right)+3.545-0.88 \log \lambda_{E} \\
& \log \left(L_{H, \mathrm{Gal}}\right)=0.86 \log \left(L_{\mathrm{bol}}\right)+4.530-0.86 \log \lambda_{E} \\
& \log \left(L_{K, \mathrm{Gal}}\right)=0.88 \log \left(L_{\mathrm{bol}}\right)+3.577-0.88 \log \lambda_{E} .
\end{aligned}
$$

The above relation is derived for the local universe. However, there is evidence that the black hole mass and host luminosity relationship evolves with redshift (Peng et al. 2006a, 2006b; Ho 2007; Decarli et al. 2010; Merloni et al. 2010; Bennert et al. 2010, 2011). Allowing for selection effects, the evolutionary trend is $M_{\mathrm{BH}} / L_{\text {sph }} \propto(1+z)^{1.4 \pm 0.2}$ (Bennert et al. 2010, 2011). As most XC413 quasars are at high redshift, we need to include this effect. We added an additional redshift term in the above Equations (4)-(7), giving the following equations:

$$
\begin{aligned}
\log \left(L_{B, \mathrm{Gal}}\right)= & 0.84 \log \left(L_{\mathrm{bol}}\right)+3.914-0.84 \log \lambda_{E} \\
& -1.18 \log (1+z)
\end{aligned}
$$

$$
\begin{aligned}
\log \left(L_{J, \mathrm{Gal}}\right)= & 0.88 \log \left(L_{\mathrm{bol}}\right)+3.545-0.88 \log \lambda_{E} \\
& -1.23 \log (1+z)
\end{aligned}
$$

$$
\begin{aligned}
\log \left(L_{H, \mathrm{Gal}}\right)= & 0.86 \log \left(L_{\mathrm{bol}}\right)+4.530-0.86 \log \lambda_{E} \\
& -1.20 \log (1+z)
\end{aligned}
$$

$$
\begin{aligned}
\log \left(L_{K, \mathrm{Gal}}\right)= & 0.88 \log \left(L_{\mathrm{bol}}\right)+3.577-0.88 \log \lambda_{E} \\
& -1.23 \log (1+z) .
\end{aligned}
$$

We choose the rest-frame $J$-band luminosity $L_{J \text {,gal }}$, because this is the band closest to $1 \mu \mathrm{m}$, where the galaxy contributes strongly. The rest-frame $J$ band is also located on the flat part of the host templates, so the normalization is insensitive to uncertainties in the template slope, in contrast to the observed F814W band for quasars at redshift 1-2.

Note that, in these formulae, $L_{\text {bol }}$ has the same coefficient as $\lambda_{E}$, thus the host galaxy luminosity is physically a function of the black hole mass only (see Marconi \& Hunt 2003 for details). To apply these formulae, black hole mass estimates are needed. The XC413 quasars have 206 published black hole mass measurements (Trump et al. 2009b; Merloni et al. 2010), which are based on the scaling relationship between BEL FWHM and black hole mass (Vestergaard 2004). For the quasars with only zCOSMOS spectra, the black hole mass was estimated for only the ones with Mg II lines in the spectra (Merloni et al. 2010), using the calibration of McLure \& Jarvis (2002). For the rest of the sample, the BELs are located at the edge of the spectrum, so that reliable black hole mass estimates cannot be made. We calculated bolometric luminosities by directly integrating the
SED from $24 \mu \mathrm{m}$ to $40 \mathrm{keV}$ (see Paper II). The rest-frame $J$-band galaxy luminosity is then calculated using Equation (9) for the 206 objects with black hole masses, hereinafter the "XC206" sample.

We need to assume a host galaxy SED to do the corrections. We have no information on the galaxy type of the host for most of the quasars, even with the help of the HST ACS images, as the bulk of the XMM-COSMOS quasars are at $z>1$. We checked the 16 galaxy templates from the SWIRE template library (Polletta et al. 2007). We normalized all the template to the rest-frame $J$ band in Figure 17. We find that none of our quasars show obvious polycyclic aromatic hydrocarbon (PAH) feature in the mid-infrared, which is a prominent feature of starburst galaxy spectra. This could, however, be due to the gap in photometry coverage between the Spitzer bands. From the Spitzer photometry alone, it is difficult to detect such features. Starburst galaxy templates with a prominent PAH feature are not suitable for host correction, though, as the PAH feature could exceed the observed (interpolated) SED. The elliptical and spiral galaxies have similar SED shapes from 0.4-4 $\mu \mathrm{m}$, with a dispersion less than 0.1 dex. This wavelength range is where the majority of the host galaxy contribution lies. We checked all spiral or elliptical galaxy templates and the correcting results are similar to each other. Therefore, for the purpose of host correction, it makes no difference which templates to choose.

We used a 5 Gyr old elliptical galaxy template (Polletta et al. 2007 ) normalized to the rest-frame $J$-band galaxy luminosity, and subtracted the galaxy contribution from the observed SED. An example is shown in Figure 18. In this case, the observed SED is rather flat. After subtracting a scaled host contribution (blue dashed line), the corrected SED (magenta solid line) shows a shape close to that of E94 (red dashed line).

This subtraction is successful in 203 cases in XC206. We have an oversubtraction problem in just three cases $(1.46 \%)$, and two out of the three are oversubtracted by less than 0.1 dex. By oversubtraction, we mean the normalized host galaxy SED exceeds the observed SED at a certain wavelength range (usually at $\sim 1 \mu \mathrm{m}$, where the galaxy contributes the most). If we use the local scaling relationship directly, i.e., without the redshift correction, there would be 34 quasars in which the estimated host flux exceeds the observed flux, leading to larger oversubtraction problem. This suggests that the evolutionary term is needed, and gives some support to the idea that the $M-\sigma$ relation evolves. The oversubtraction could be due either to dispersion or evolution of the scaling relationship. On the other hand, undersubtraction also happens in the correction, which is more difficult to classify. By undersubtraction, we mean that the correction leaves a flat SED at around $1 \mu \mathrm{m}$.

The host correction based on the scaling relationship we applied here is probably not the most accurate, but is the best available at present. We applied this host galaxy correction for these 203 quasars, hereinafter "XC203." The XC203 quasars are plotted as green hexagons in Figures 2-5. In this subsample, four $^{38}$ are radio-loud according to the criteria discussed in Section 3.3. We calculated the mean SED and dispersion SED as the previous section.

The mean and dispersion of the XC203 SEDs after all the corrections, including galaxy correction, resembles the E94 mean SED (Figures 19 and 20).

In Figure 21, we compared the mean XC203 SED with other mean SEDs: E94 radio-quiet, R06, Hopkins et al. (2007)

\footnotetext{
$38 \mathrm{XID}=5230,5275,5517,54541$.
} 


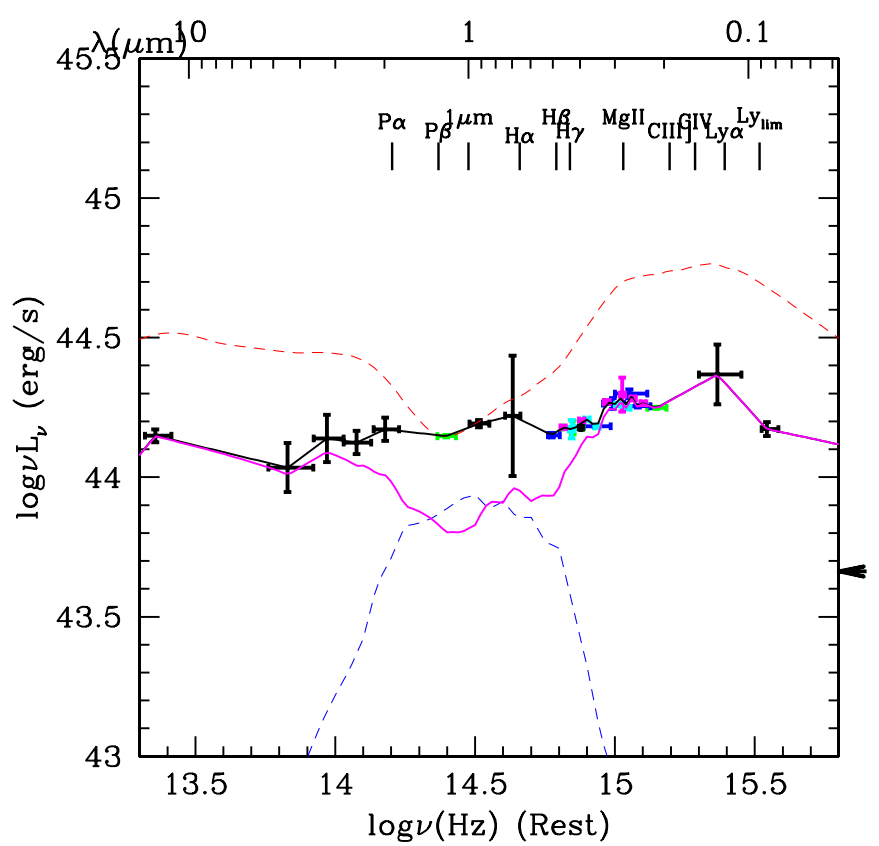

Figure 18. SED of source COSMOS_J150.50924+2.699418 (XID = 86) at $z=0.794$ ( $i$-band absolute magnitude -22.4 ). The mass of the black hole is $\log M_{\mathrm{BH}}=8.24$. The black solid line show the SED as the interpolation of the observed data. The magenta solid line shows the SED after host galaxy correction. Red dashed line = E94 RQ mean SED. Blue dashed line = host galaxy template (5Gyr elliptical galaxy) normalized at $L_{J, \text { Gal }}$ calculated from the Marconi \& Hunt (2003) scaling relationship adding the evolutionary term. The points are color coded as in Figure 8.

(A color version of this figure is available in the online journal.)

and the Shang et al. (2011). The R06 SEDs used a "gaprepair" technique, which replaces the missing values with the normalized E94 mean SED to the adjacent photometry bands. As R06 has limited coverage in near-infrared $J, H$, and $K$ bands ( $J: 40 / 259, H: 35 / 259, K: 42 / 259)$, the mean SED is therefore, by construction, similar to the E94 radio-quiet mean SED. Hopkins et al. (2007) just combined the R06 mean SED template with the composite quasar SED (Vanden Berk et al. 2001), and so their SED template has a similar shape as R06. Assef et al. (2010) did the SED fitting from $0.03 \mu \mathrm{m}$ to $30 \mu \mathrm{m}$ for the NOAO DeepWide Field Survey Boötes field and got a converged AGN SED template quite similar to R06 (using R06 as a starting point). As in this study, the AGN is sectected using a broad range of methods, the template is not exclusively applicable to type 1 AGN, so we do not plot the Assef et al. (2010) template in Figure 21. The Shang et al. (2011) mean SED is calculated using nearby bright quasars, which have the same selection bias as E94 and so finds a similar shape to E94. Compared to all these SED templates, the XC203 mean SED is flatter due to possible excess host contribution, not corrected because of the dispersion in the scaling relationship itself.

\subsection{The Variety of Type 1 AGN SEDs in COSMOS}

Simply using the dispersion in the SEDs (Figure 16) does not give a full picture of the variety of SEDs in the XC413 sample. To illustrate the variety of type 1 AGN SEDs found in the XMMCOSMOS sample, we have selected four examples that span the range of properties (Figures 22, Sources A, B, C, D). The four sources are also marked in Figures 2-5. These four examples illustrate:

1. Source A. "Normal" SED, with photometry points lying strikingly close to the E94 radio-quiet mean SED (red dashed line), except for the few that are clearly affected by the BELs, $\mathrm{H} \alpha, \mathrm{CIV}$, and $\mathrm{Ly} \alpha$, and the point beyond the Lyman limit.

2. Source B. "Reddened" SED, with an optical/UV SED too blue to be a host galaxy, but dropping rapidly toward the $\mathrm{UV}$, an effect quite likely caused by reddening. The $\sim 1 \mathrm{dex}$ drop in the $u$ band compared to the E94 RQ mean would correspond to a fairly modest extinction of $E(B-V)=$ 0.18, for an SMC extinction curve (Gordon et al. 2003).

3. Source C. "Host-dominated" SED, matching closely to galaxy templates. The observed SED could be fitted as a strong galaxy plus a faint AGN component. About $10 \%$
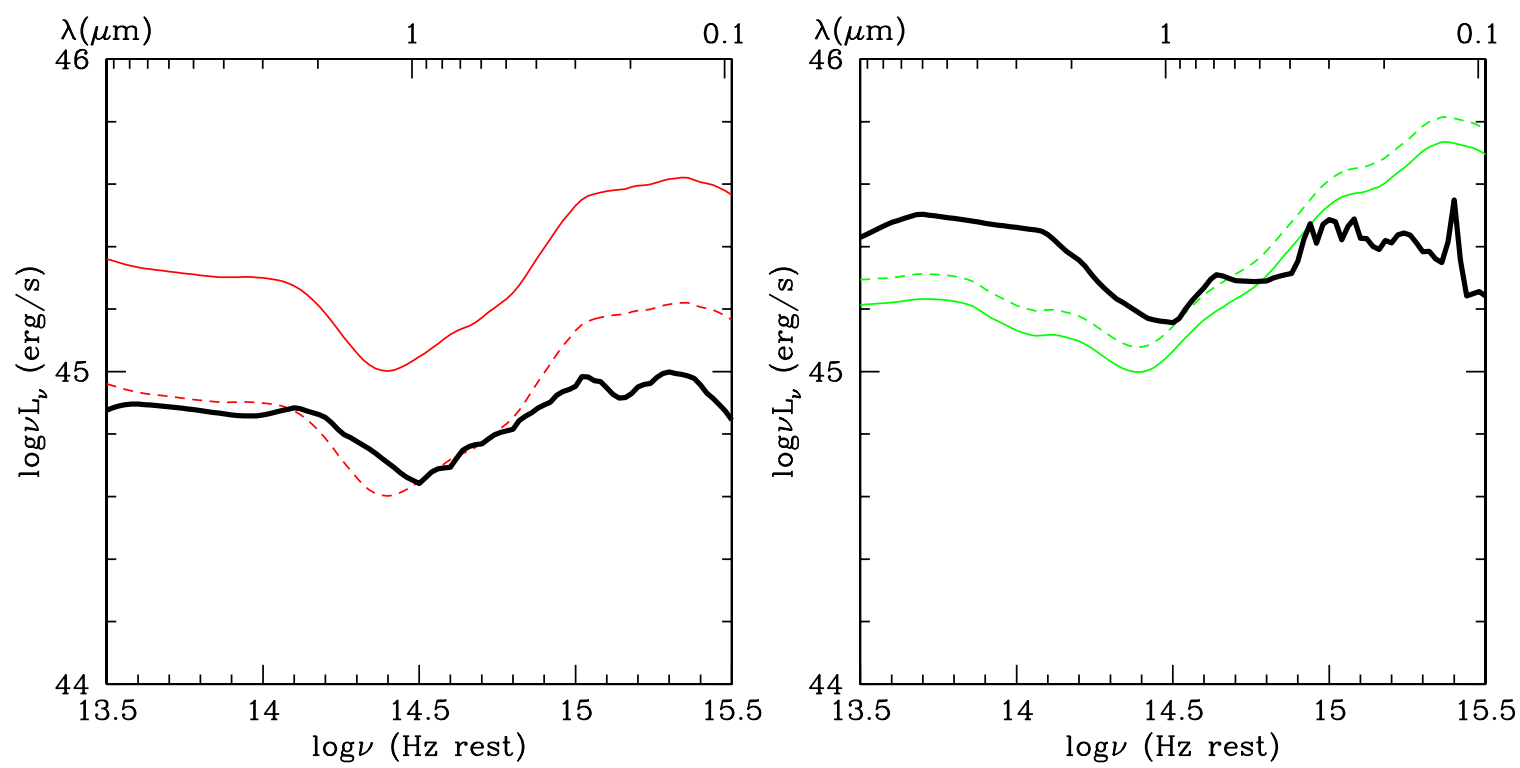

Figure 19. Left: the mean IR to UV SED for 199 radio-quiet (RQ) sources in our sample after all corrections including host galaxy subtraction (black solid line). The red lines are the E94 radio-quiet mean SED (the dashed line is normalized to the XMM-Newton sample). Right: the mean IR to UV SED for four radio-loud (RL) quasars after all corrections (black solid line). The green lines are the E94 radio-loud mean SEDs (dashed is normalized to the XMM-Newton sample).

(A color version of this figure is available in the online journal.) 

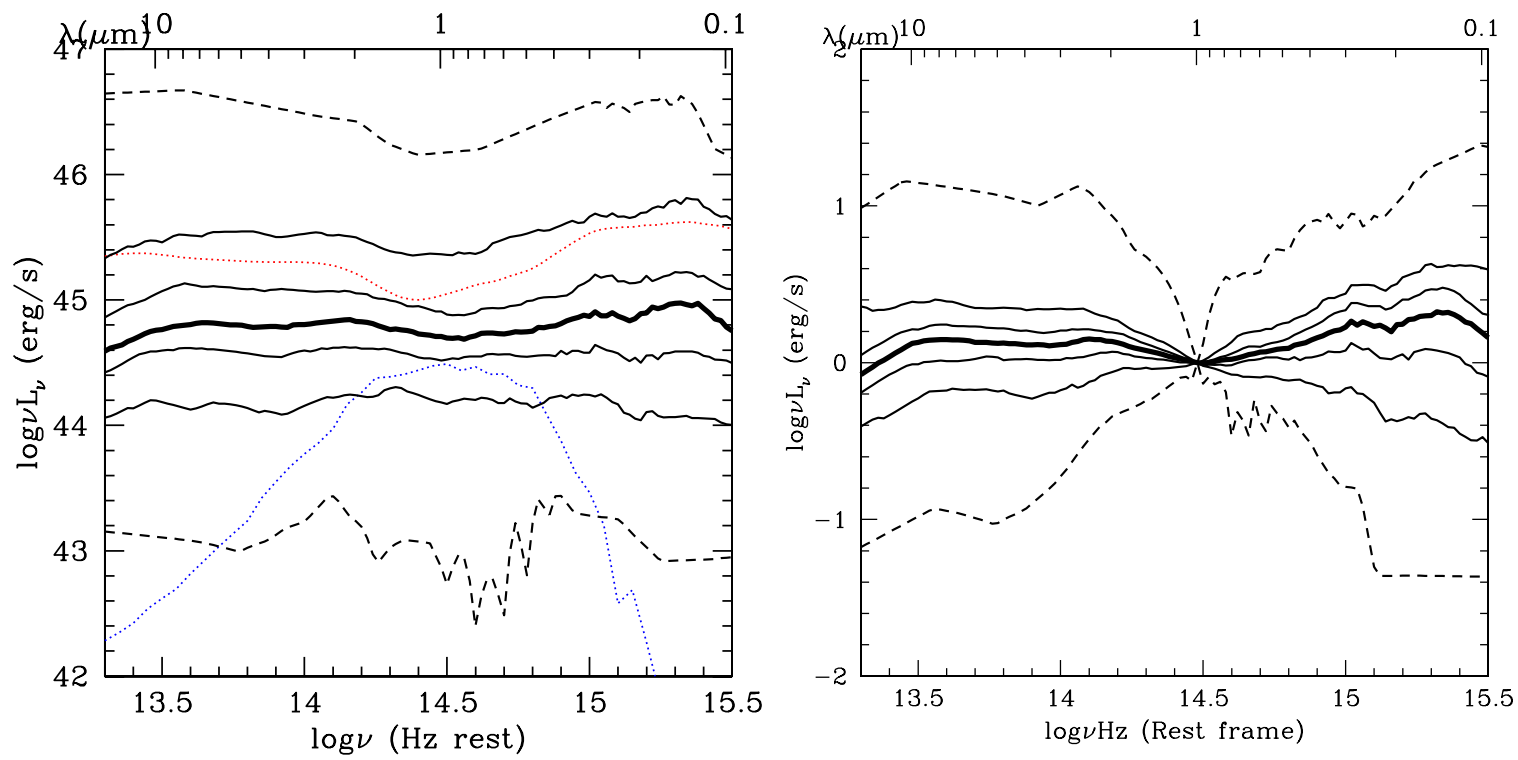

Figure 20. Median (thick black line) SED and the 68\%, 90\% (thin solid black lines), and 100\% (dashed black lines) percentile envelopes in the ultraviolet to infrared $(0.9 \mu \mathrm{m}-24 \mu \mathrm{m})$ range after all correction including the host galaxy subtraction: before normalization (left); normalized at $1 \mu \mathrm{m}$ (right). Red line $=$ E94 SED. Blue dotted line $=5$ Gyr elliptical galaxy template (El15).

(A color version of this figure is available in the online journal.)

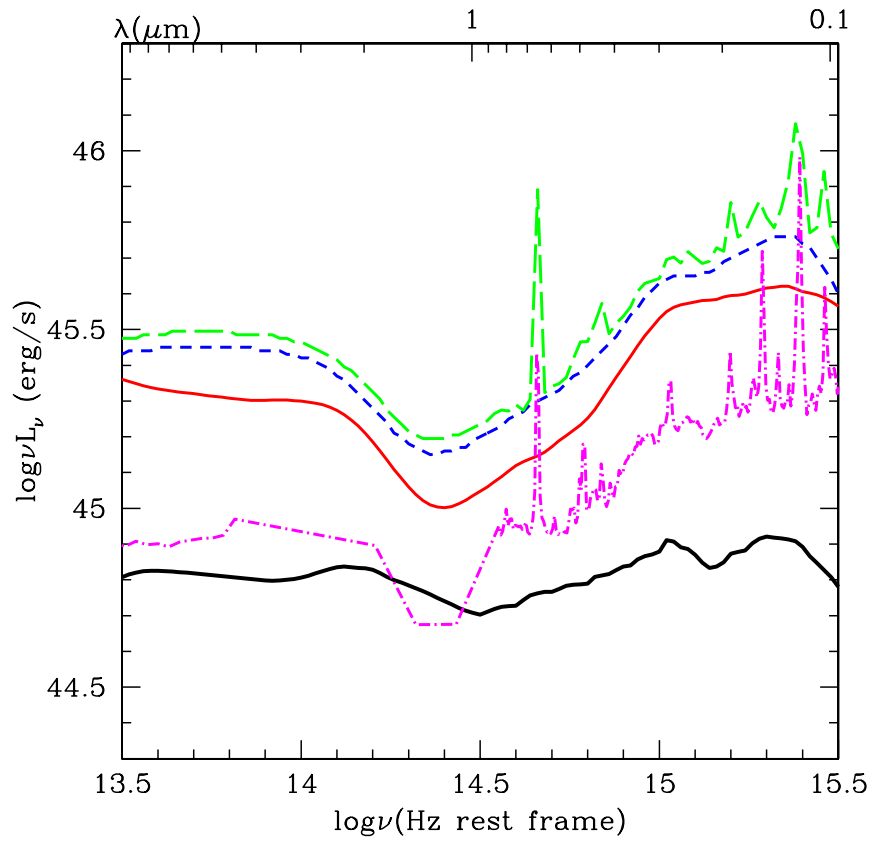

Figure 21. Comparison of the mean host-corrected SED for the 199 radioquiet quasars of XC203 (black solid line) with previous studies. The red solid line shows the E94 radio-quiet mean SED. The blue short dashed line shows the Richards et al. (2006) mean SED. The green long dashed line shows the Hopkins et al. (2007) AGN SED template. The magenta dot-dashed line shows the Shang et al. (2011) mean SED.

(A color version of this figure is available in the online journal.)

of the quasars in the sample have host contribution of more than $85 \%$ at $\sim 1 \mu \mathrm{m}$.

4. Source D. "Hot-dust-poor" SED, lacking a $1 \mu \mathrm{m}$ inflection point, but with a normal E94-like strong big blue bump. About $10 \%$ of the quasars in the sample belong to this category. These quasars have been discussed in detail in Hao et al. (2010).
As the variety of the SED shapes in the sample is a continuous distribution, the fraction of the sources of the above four types depend on how we define the selection criteria for each type. Besides, for most of the sources, the SED shape can be explained by combining two or more of the above four types. So, the fraction of the sources in each type can not be easily estimated.

Three of these four types have been seen before in AGNs at lower redshift, but similar luminosity. The early $2-10 \mathrm{keV}$ sky surveys (e.g., Ariel V, Cooke et al. 1978, HEAO-1 A2, Piccinotti et al. 1982) produced samples of a few dozen AGNs that have similar SED characteristics. Ward et al. (1987) compiled $U$ band to IRAS $100 \mu \mathrm{m}$ SEDs for the "Piccinotti" AGN and divided them into three types based on their $f(60 \mu \mathrm{m}) / f(12 \mu \mathrm{m})$ to $f(1.2 \mu \mathrm{m}) / f(0.36 \mu \mathrm{m})$ flux ratios: (1) prominent big blue bump objects (e.g., 3C 273), which correspond to the "Normal," E94like, objects; (2) rapidly dropping optical SEDs with no strong FIR emission (e.g., MCG-6-30-15), which are reddened AGNs and resemble the "No Big Bump" objects; (3) strong FIR, weak optical/UV objects (e.g., NGC 3227), which are dominated by host galaxy emission in both regions and so correspond to the "Host-dominated" objects. The host FIR emission in Class C AGNs is often extended and lies in the range of normal galaxies in $50 \%$ of cases, and of starbursts in the rest.

In a companion paper (Carleton et al. 1987) the same authors argued that all of these types were consistent with a single intrinsic form of the quasar SED, modified only by obscuration and host galaxy contamination. The bluest of the Class A objects would exhibit this SED form, which is close to the E94 mean SED. Many of the "reddened Class B" objects have X-ray column densities $N_{\mathrm{H}} \sim 10^{22}-10^{23} \mathrm{~cm}^{-2}$, and have intermediate AGN types (1.5, 1.8, 1.9; Osterbrock \& Koski 1976) indicating modest reddening of the BEL region and continuum of $A_{V} \sim$ $0.5-3$. We do not have $\mathrm{H} \alpha / \mathrm{H} \beta$ ratios, or X-ray $N_{\mathrm{H}}$ values, for more than a handful of the COSMOS type 1 AGN sample and so cannot yet test whether the XMM-COSMOS sample shares these properties with the Piccinotti AGN. We explore the AGNhost-reddening parameter space in detail in Paper III. 

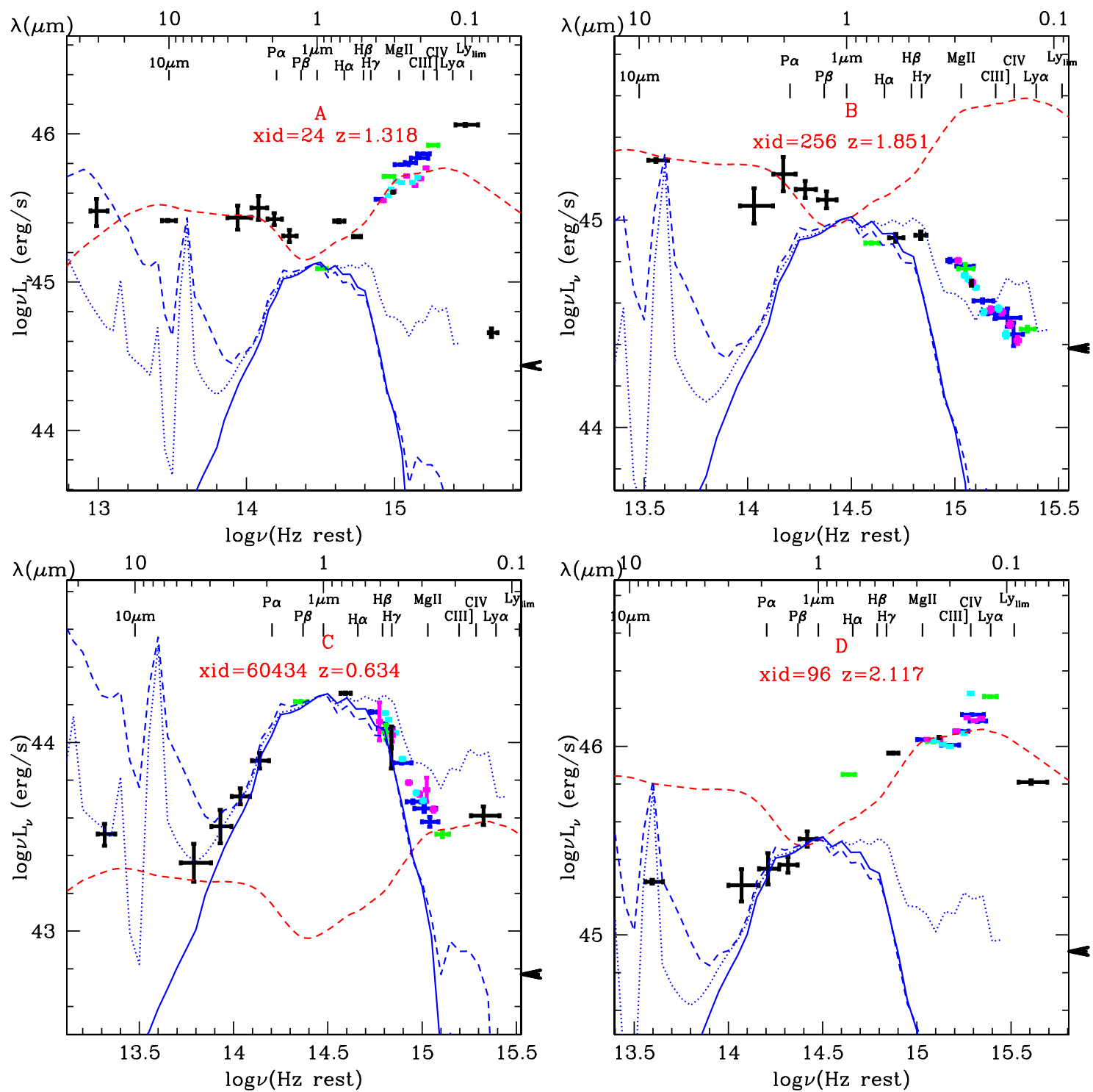

Figure 22. Extreme examples of SEDs: top left (Source A) a close analog of the E94 mean SED; top right (Source B) no big blue bump, probably due to reddening and/or a strong galaxy component; bottom left (Source C) a probable galaxy-dominated AGNs; bottom right (Source D) strong big blue bump but no $1 \mu \mathrm{m}$ inflection due to a weak near-IR bump. The data points and the E94 RQ SEDs are shown as in Figure 8. The galaxy templates are shown as in Figure 14 normalized at $1 \mu \mathrm{m}$.

(A color version of this figure is available in the online journal.)

The fourth class, D, "hot-dust-poor," has not been recognized before. The missing inflection point is apparently due to the reduced "hot dust" bump by at least a factor 3 in the near-IR. As the hot dust emission is attributed to the "torus" invoked in unified models of AGNs to explain the type 1/type 2 dichotomy, the existence of a class of AGNs without this "torus" emission raises questions about the universality of the unified model. This class of sources are discussed in detail in Hao et al. (2010) for COSMOS and Hao et al. (2011) for optically selected samples.

\section{SUMMARY AND CONCLUSIONS}

In this paper we have assembled a large sample of 413 type 1 AGNs (emission line FWHM $>2000 \mathrm{~km} \mathrm{~s}^{-1}$ ), selected in Xrays by XMM-Newton within the COSMOS field. The sample includes sources with spectroscopic redshifts and a uniform multiwavelength coverage from the X-ray to the far-infrared, with $33.6 \%$ radio (VLA) coverage too.

We briefly analyzed the optical and X-ray properties of the sample with the main aim to compare them with the Elvis et al.
(1994) sample, which is our reference for the construction of the mean SED.

The main goal of this paper is to derive the mean type 1 AGN SED. We have derived SEDs for all the 413 type 1 AGNs. For 203 sources, we could use the black hole mass and the $M_{\mathrm{BH}}-M_{\text {bulge }}$ scaling relation to produce host-subtracted SEDs. The COSMOS type 1 AGN sample spans a much larger range of redshift and luminosity than the E94 sample. Only six of these AGNs, $\sim 1.5 \%$, are radio-loud.

These SEDs make use of the vast COSMOS photometric data set, and so contain many photometric points (a mean of 35 per SED). The SEDs are especially well sampled in the optical/UV band $(\sim 0.1-1 \mu \mathrm{m})$, with a mean of 18 photometric points. We have corrected the SEDs for Galactic extinction, have restricted the data collection time interval to limit variability, and have made a correction for the BEL contribution. The SEDs were re-sampled on a uniform rest-frame frequency grid.

The mean SED in the rest-frame $8 \mu \mathrm{m}$ to $4000 \AA$ are calculated based on detections only. Mean SEDs beyond this range are calculated based on a reasonable power-law assumption. 
The mean SED of the sample before host galaxy correction is quite different from the E94 mean radio-quiet SED and does not show a prominent $1 \mu \mathrm{m}$ inflection point between the UV and near-IR bumps. Subsamples of AGNs (with black hole mass estimation) corrected for host galaxy contribution using the Marconi \& Hunt (2003) scaling relationship, with an additional redshift evolution term, restores the $1 \mu \mathrm{m}$ inflection to the mean COSMOS type 1 SED. This shows that host galaxy contamination is likely to be a major contributor to the variety of SEDs in an $\mathrm{X}$-ray-selected sample. Evolution of the $M-\sigma$ relation is supported by the better host subtraction obtained using the Bennert et al. (2011) relation.

The SEDs before and after the corrections for the Galactic extinction, BEL contributions, constrained variability, and host galaxy contribution are available in online journal.

Both host contamination and reddening for all COSMOS quasars will be addressed in companion papers (Hao et al. 2012a, 2012b).

Several extreme types of AGN SED were identified, corresponding to an E94 SED, a reddened E94 SED, and a hostdominated SED. These three SED types have been seen previously (e.g., Ward et al. 1987). A new subclass of "hot-dustpoor" quasars has been found that appears to lack strong hot dust emission. These will be of interest for unification models as they appear to indicate the absence of the standard obscuring "torus." Their properties are discussed in Hao et al. (2010, 2011).

The COSMOS AGN photometry coverage continues to expand. In particular, the far-infrared to millimeter region: far-IR (60-600 $\mu \mathrm{m})$ has been observed with the Herschel satellite, SCUBA2 will image the field at $850 \mu \mathrm{m}$, and $1.1 \mathrm{~mm}$ imaging of much of the field by AzTeC has been performed and should yield several hundred sources (Scott et al. 2008; Austermann et al. 2009). The quasar SED properties over wider wavelength ranges will be possible in the near future.

M.E. and H.H. thank J. McDowell for useful discussion. This work was supported in part by NASA Chandra grant number GO7-8136A (H.H., F.C., M.E.), NASA contract NAS8-39073 (Chandra X-ray Center), and the Smithsonian Scholarly Studies (F.C.). K.J. acknowledges support from the Emmy Noether Programme of the German Science Foundation (DFG) through grant number JA $1114 / 3-1$. In Italy this work is supported by ASI/INAF contracts I/009/10/0, I/024/05/0, and I/088/06. In Germany this project is supported by the Bundesministerium für Bildung und Forschung/Deutsches Zentrum für Luft und Raumfahrt and the Max Planck Society. M.S. and G.H. acknowledge support bei the Leibniz Prize of the Deutsche Forschungagemeinschaft, DFG (HA 1850/28-1). Y.L. acknowledges partial funding support by the Directional Research Project of the Chinese Academy of Sciences under project No. KJCX2-YW-T03 and by the National Natural Science Foundation of China under grant Nos. 10821061, 10733010, 10725313, and by 973 Program of China under grant 2009CB824800.

\section{REFERENCES}

Abazajian, K. N., Adelman-McCarthy, J. K., Agüeros, M. A., et al. 2004, AJ, 128,502

Abazajian, K. N., Adelman-McCarthy, J. K., Agüeros, M. A., et al. 2009, ApJS, 182,543

Appleton, P. N., Fadda, D. T., Marleau, F. R., et al. 2004, ApJS, 154, 147

Arnaud, K. A. 1996, in ASP Conf. Ser. 101, Astronomical Data Analysis Software and Systems V, ed. G. H. Jacoby \& J. Barnes (San Francisco, CA: ASP), 17
Assef, R. J., Kochanek, C. S., Brodwin, M., et al. 2010, ApJ, 713, 970 Austermann, J. E., Aretxaga, I., Hughes, D. H., et al. 2009, MNRAS, 393, 1573 Baldwin, J. A. 1977, ApJ, 214, 679

Bennert, V. N., Auger, M. W., Treu, T., Woo, J., \& Malkan, M. A. 2011, ApJ, 742, 107

Bennert, V. N., Treu, T., Woo, J.-H., et al. 2010, ApJ, 708, 1507

Brissenden, R. J. V. 1989, PhD thesis, Australian National University

Brusa, M., Comastri, A., Daddi, E., et al. 2005, A\&A, 2005, 421, 69

Brusa, M., Zamorani, G., Comastri, A., et al. 2007, ApJS, 2007, 172, 353

Brusa, M., et al. 2010, ApJ, 716, 348

Capak, P., Aussel, H., Ajiki, M., et al. 2007, ApJS, 172, 99

Cappelluti, N., et al. 2009, A\&A, 497, 635

Cardelli, J. A., Clayton, G. C., \& Mathis, J. S. 1989, ApJ, 345, 245

Carleton, N. P., Elvis, M., Fabbiano, G., et al. 1987, ApJ, 318, 595

Ciliegi, P., Vignali, C., Comastri, A., et al. 2003, MNRAS, 342, 575

Cirasuolo, M., McLure, R. J., Dunlop, J. S., et al. 2007, MNRAS, 380, 585

Civano, F., Elvis, M., Brusa, M., et al. 2012, ApJS, 201, 30

Cooke, B. A., Ricketts, M. J., Maccacaro, T., et al. 1978, MNRAS, 182, 489

Decarli, R., Falomo, R., Treves, A., et al. 2010, MNRAS, 402, 2453

Dickey, J. M., \& Lockman, F. J. 1990, ARA\&A, 28, 215

Donley, J. L., Koekemoer, A. M., Brusa, M., et al. 2012, ApJ, 748, 142

Dunne, L., \& Eales, S. A. 2001, MNRAS, 327, 697

Elvis, M., Civano, F., Vignali, C., et al. 2009, ApJS, 184, 158

Elvis, M., Wilkes, B. J., McDowell, J. C., et al. 1994, ApJS, 95, 1

Eskridge, P. B., Fabbiano, G., \& Kim, Dong-Woo 1995, ApJ, 448, 70

Falter, S., et al. 2004, in Proc. IAU Colloq. 195, Outskirts of Galaxy Clusters: Intense Life in the Suburbs, ed. A. Diaferio (Cambridge: Cambridge Univ. Press), 233

Ferland, G. J., \& Osterbrock, D. E. 2005, Astrophysics of Gaseous Nebulae and Active Galactic Nuclei (Mill Valley, CA: Univ. Science Books)

Frayer, D. T., Sanders, D. B., Surace, J. A., et al. 2009, AJ, 138, 1261

Gabor, J. M., Impey, C. D., Jahnke, K., et al. 2009, ApJ, 691, 705

Goldschmidt, P., Kukula, M. J., Miller, L., \& Dunlop, J. S. 1999, ApJ, 511, 612

Gordon, K., Clayton, G. C., Misselt, K. A., Landolt, A. U., \& Wolff, M. J. 2003, ApJ, 594, 279

Green, P., Forster, K., \& Kuraszkiewicz, J. 2001, ApJ, 556, 727

Hao, H., Elvis, M., Civano, F., et al. 2010, ApJ, 724, L59

Hao, H., Elvis, M., Civano, F., \& Lawrence, A. 2011, ApJ, 733, 108

Hao, H., et al. 2012a, MNRAS, submitted

Hao, H., et al. 2012b, MNRAS, submitted

Hao, H., et al. 2012c, ApJL, submitted

Hasinger, G., Cappelluti, N., Brunner, H., et al. 2007, ApJS, 172, 29

Hawkins, S. E. 2007, A\&A, 462, 581

Ho, L. C. 2007, ApJ, 669, 821

Hopkins, P. F., Hernquist, L., Cox, T. J., et al. 2006, ApJS, 163, 1

Hopkins, P. F., Richards, G. T., \& Hernquist, L. 2007, ApJ, 654, 731

Ilbert, O., Capak, P., Salvato, M., et al. 2009, ApJ, 690, 1236

Ivezić, Ž. 2004, in Multiwavelength AGN Surveys, ed. R. Maiolino \& R. Mújica (Singapore: World Scientific), 53

Ivezić, Ž, Menou, K., Knapp, G. R., et al. 2002, AJ, 124, 2364

Jester, S., Schneider, D. P., Richards, G. T., et al. 2005, AJ, 130, 873

Jiang, L., Fan, X., Ivezić, Ž., et al. 2007, ApJ, 656, 680

Kartaltepe, J., Sanders, D. B., Le Floc'h, E., et al. 2010, ApJ, 709, 572

Kellermann, K. I., Sramek, R., Schmidt, M., Shaffer, D. B., \& Green, R. 1989, AJ, 98, 1195

Koekemoer, A. M., Aussel, H., Calzetti, D., et al. 2007, ApJS, 172, 196

Komatsu, E., Dunkley, J., Nolta, M. R., et al. 2009, ApJS, 180, 330

Kuraszkiewicz, J. K., Wilkes, B. J., Hooper, E. J., et al. 2003, ApJ, 590, 128

Laor, A., Fiore, F., Elvis, M., Wilkes, B. J., \& McDowell, J. C. 1997, ApJ, 477,93

Lapi, A., González-Nuevo, J., Fan, L., et al. 2011, ApJ, 742, 24

Le Floc'h, E., Aussel, H., Ilbert, O., et al. 2009, ApJ, 703, 222L

Lilly, S. J., Le Févre, O., Renzini, A., et al. 2007, ApJS, 172, 70

Lilly, S. J., Le Brun, V., Maier, C., et al. 2009, ApJS, 184, 218

Luo, B., Brandt, W. N., Xue, Y. Q., et al. 2010, ApJS, 187, 560

Lusso, E., Comastri, A., Vignali, C., et al. 2010, A\&A, 512, 34

Magorrian, J., Tremaine, S., Richstone, D., et al. 1998, AJ, 115, 2285

Mainieri, V., Hasinger, G., Cappelluti, N., et al. 2007, ApJS, 172, 368

Marchese, E., Della Ceca, R., Caccianiga, A., et al. 2012, A\&A, 539, 48

Marconi, A., \& Hunt, L. K. 2003, ApJ, 589, L21

Marconi, A., Risaliti, G., Gilli, R., et al. 2004, MNRAS, 351, 169

McCracken, H. J., Capak, P., Salvato, M., et al. 2010, ApJ, 708, 202

McCracken, H. J., Peacock, J. A., Guzzo, L., et al. 2007, ApJS, 172, 314

McLeod, K. K., \& Rieke, G. H. 1994, ApJ, 431, 137

McLure, R. J., \& Jarvis, M. J. 2002, MNRAS, 337, 109

Merloni, A., Bongiorno, A., Bolzonella, M., et al. 2010, ApJ, 708, 137

Oke, J. B., \& Gunn, J. E. 1983, ApJ, 266, 713 
Osterbrock, D. E., \& Koski, A. T. 1976, MNRAS, 176, 61

Peng, C. Y., Impey, C. D., Ho, L. C., Barton, E. J., \& Rix, H. 2006a, ApJ, 640, 114

Peng, C. Y., Impey, C. D., Rix, H.-W., et al. 2006b, ApJ, 649, 616

Peterson, B. M. 1997, An Introduction to Active Galactic Nuclei (Cambridge: Cambridge Univ. Press)

Piccinotti, G., Mushotzky, R. F., Boldt, E. A., et al. 1982, ApJ, 253, 485

Polletta, M., Courvoisier, T. J.-L., Hooper, E. J., \& Wilkes, B. J. 2000, A\&A, 362,75

Polletta, M., Tajer, M., Maraschi, L., et al. 2007, ApJ, 663, 81

Puchnarewicz, E. M., Mason, K. O., Carrera, F. J., et al. 1997, MNRAS, 291, 177

Richards, G. T., Lacy, M., Storrie-Lombardi, L. J., et al. 2006, ApJS, 166, 470

Risaliti, G., \& Elvis, M. 2004, in Supermassive Black Holes in the Distant Universe, ed. A. J. Barger (ASSL Vol. 308; Dordrecht: Kluwer), 187

Rosati, P., Tozzi, P., Giacconi, R., et al. 2002, ApJ, 566, 667

Salvato, M., Hasinger, G., Ilbert, O., et al. 2009, ApJ, 690, 1250

Sanders, D. B., Salvato, M., Aussel, H., et al. 2007, ApJS, 172, 86

Schinnerer, E., Sargent, M. T., Bondi, M., et al. 2010, ApJS, 188, 384

Schinnerer, E., Smolčić, V., Carilli, C. L., et al. 2007, ApJS, 172, 46

Schlegel, D. J., Finkbeiner, D. P., \& Davis, M. 1998, ApJ, 500, 525

Schneider, D. P., Hall, P. B., Richards, G. T., et al. 2007, AJ, 130, 367

Scott, K. S., Austermann, J. E., Perera, T. A., et al. 2008, MNRAS, 385, 2225

Scoville, N. Z., Abraham, R. G., Aussel, H., et al. 2007a, ApJS, 172, 38
Scoville, N. Z., Aussel, H., Brusa, M., et al. 2007b, ApJS, 172, 1

Sergeev, S. G., Doroshenko, V. T., Golubinskiy, Yu. V., Merkulova, N. I., \& Sergeeva, E. A. 2006, in ASP Conf. Ser. 360, AGN Variability from X-Rays to Radio Waves, ed. C. M. Gaskell et al. (San Francisco, CA: ASP), 13

Shang, Z., Brotherton, M. S., Wills, B. J., et al. 2011, ApJS, 196, 2

Shang, Z., Wills, B. J., Wills, D., \& Brotherton, M. S. 2007, AJ, 134, 294

Stocke, J. T., Morris, S. L., Weymann, R. J., \& Foltz, C. B. 1992, ApJ, 396, 487

Sutherland, W., \& Saunders, W. 1992, MNRAS, 259, 413

Taniguchi, Y., Scoville, N., Murayama, T., et al. 2007, ApJS, 172, 9

Terashima, Y., \& Wilson, A. S. 2003, ApJ, 583, 145

Tremaine, S., Gebhardt, K., Bender, R., et al. 2002, ApJ, 574, 740

Trichas, M., Green, P. J., Silverman, J. D., et al. 2012, ApJS, 200, 17

Trump, J. R., Impey, C. D., Elvis, M., et al. 2009a, ApJ, 696, 1195

Trump, J. R., Impey, C. D., Kelly, B. C., et al. 2009b, ApJ, 700, 49

Trump, J. R., Impey, C. D., Kelly, B. C., et al. 2011, ApJ, 733, 60

Trump, J. R., Impey, C. D., McCarthy, P. J., et al. 2007, ApJS, 172, 383

Urry, C. M., \& Padovani, P. 1995, PASP, 107, 803

Vanden Berk, D. E., Richards, G. T., Bauer, A., et al. 2001, AJ, 122, 549

Vanden Berk, D. E., Shen, J., Yip, C.-W., et al. 2006, AJ, 131, 84

Vanden Berk, D. E., Wilhite, B. C., Kron, R. G., et al. 2004, ApJ, 601, 692

Vestergaard, M. 2004, ApJ, 601, 676

Ward, M. J., Elvis, M., \& Fabbiano, G. 1987, ApJ, 315, 74

Wilkes, B. J., \& Elvis, M. 1987, ApJ, 323, 243

Zamojski, M. A., Schiminovich, D., Rich, R. M., et al. 2007, ApJS, 172, 468 